\title{
Does a Randall-Sundrum Brane World Effective Potential Influence Axion Walls Helping to Form a Cosmological Constant Affecting Inflation?
}

\author{
Andrew Walcott Beckwith \\ Chongqing University Huxi Campus, Chongqing, China \\ Email: Rwill9955b@gmail.com, abeckwith@uh.edu \\ Received 8 December 2015; accepted 26 January 2016; published 29 January 2016 \\ Copyright (C) 2016 by author and Scientific Research Publishing Inc. \\ This work is licensed under the Creative Commons Attribution International License (CC BY). \\ http://creativecommons.org/licenses/by/4.0/

(c) (i) Open Access

\section{Abstract}

In 2003, Guth posed the following question in a KITP seminar in UCSB. Namely "Even if there exist $10^{1000}$ vacuum states produced by String theory, does inflation produce overwhelmingly one preferred type of vacuum states over the other possible types of vacuum states"? This document tries to answer how a preferred vacuum state could be produced, and by what sort of process. We construct a di quark condensate leading to a cosmological constant in line with known physical observations. We use a phase transition bridge from a tilted washboard potential to the chaotic inflationary model pioneered by Guth which is congruent with the slow roll criteria. This permits criteria for initiation of graviton production from a domain wall formed after a transition to a chaotic inflationary potential. It also permits investigation of if or not axion wall contributions to inflation are necessary. If we reject an explicit axion mass drop off to infinitesimal values at high temperatures, we may use the Bogomolnyi inequality to rescale and reset initial conditions for the chaotic inflationary potential. Then the Randall-Sundrum brane world effective potential delineates the end of the dominant role of di quarks, and the beginning of inflation. And perhaps answers Freeman Dysons contention that Graviton production is unlikely given present astrophysical constraints upon detector systems. We end this with a description in the last appendix entry, Appendix VI, as to why, given the emphasis upon di quarks, as to the usefulness of using times before Planck time interval as to modeling our physical system and its importance as to emergent field structures used for cosmological modeling.

\section{Keywords}

Branes, Axion Walls, Bogomolnyi Inequality, Four- and Five-Dimensional Cosmological Constant 


\section{Introduction}

It is well known through conventional calculations via QCD that there is a huge disconnection between what is calculated for a cosmological constant [1], and the de facto observed phenomenological data which purports to support a cosmological constant far lower in magnitude than what is presumed to be an artifact of vacuum polarization [2]. This paper in part is an answer to this odd divergence and a summary of a different tack to giving a bound to the cosmological constant problem than one supplied by conventional applications of QCD. I contend that not only a difference would take on the cosmological constant yield dividends, but it would also yield an experimentally falsifiable model as to graviton production, as an answer to Freeman Dysons challenge to the astrophysics community to come up with detector schemes realistically capable of detecting spin two gravitons with our present level of detector technology.

As a general point, when we say S.H.O. I am referring to the acronym of simple harmonic oscillator. This paper is dedicated to physics way beyond the S.H.O. (simple harmonic oscillator) approximation and is dedicated to the premise that nonlinear contributions, which are not fully treated in the conventional literature are of decisive importance and this paper is a first attempt to include in effects which are often neglected for the same of convenience.

Note also that an acronym WKB is referred to as a semi-classical tunneling approximation in this paper. Semi-classical approximations abound all over physics, and what we are saying is that the construction we are emphasizing allows us to incorporate more quantum mechanical procedures in our treatment of cosmology. The reason for refinements of our protocol about tunneling, as in the initial phases of cosmology lies in our treatment of what is known about zero point fluctuations, which will be brought up next. We in looking at quantum tunneling, as in the WKB idea, and it is a way to form vacuum states, initially, in cosmology and also the concept of a vacuum energy density value, which will be the reason for Equation (1) in the paper below. More about the WKB approximation will be in the $2^{\text {nd }}$ to last appendix entry of this document.

The vacuum state idea is brought up, in our document because a quantum state is said to be a vacuum state if the expectation value of the Hamiltonian in a given theory is a local minimum (the Hamiltonian of course being part of the data that defines the theory). We do not necessarily have a Hamiltonian for our modeling of cosmology, although both loop quantum gravity and Wheeler De Witt theory do refer to cosmological states, although incompletely. Needless to state, if we can define a Hamiltonian for cosmological conditions, not impossible to do, then we can go to the next idea, which is that of a vacuum energy density, which is one of the biggest problems in physics today. To a large degree, this document is an attempt to explain what are the consequences of extremely large theoretically calculated vacuum energy density values, and how to ameliorate the serious problems such calculations bring to the study of cosmology.

Zero point fluctuations of quantum fields contribute to an enormous vacuum energy density value $\rho_{\text {vacuum }}$ But the reality as seen in solar system based measurements and then in galactic astrophysics data sets have lead to a de facto observed [3]

$$
|\Lambda| \leq 10^{-56} \mathrm{~cm}^{-2} \Leftrightarrow \rho_{\text {vacuum }} \leq 10^{-29} \mathrm{~g} \cdot \mathrm{cm}^{-3}
$$

What we are doing is to give a physical model as to what could be an alternative mechanism physically to the vacuum fluctuation model used in QCD calculations to give a bound to the cosmological constant which avoids having a value roughly $10^{120}$ times too large [1]. In addition, this same value included in the cosmological constant model would help give an upper bound to graviton production in branes [4] which is a subject which will be done according to putting in the absolute magnitude of the cosmological constant in to a brane world bound to graviton production in a de Sitter, and ANTI de Sitter cosmological metric model [4] [5].

Recent field theory calculations have lowered the overshoot factor to being of the order of $10^{43}$ times larger than what is inferred experimentally [6]. This is a significant improvement, but still beggars the question as to what is really needed to get the calculated results in line with known experimental data as referred to in Equation (1) above.

The model which in the end I turned to is similar in part to what was attempted by Weinberg et al. in the early 1980s [7], with one tunneling event through a potential barrier. This was finally discarded, and what I am doing now is to in part re introduce part of the same idea but with a different scalar potential field than was attempted by Weinberg nearly two decades ago.

Dr. Abbot as of the mid 1980s attempted to lower a calculated cosmological constant value via application of 
a tilted Washboard potential for a scalar field [8]-[10]. I am using this method in part for just ONE tunneling event, plus a different constituent scalar field than used by either Weinberg or Abbot to try to get a more realistic value for the cosmological constant.

QCD is the theory of quantum chromodynamics, which is well understood. What we are doing is to try to make QCD as commensurate to quantum gravity, and quantum gravity via the idea of use of di quarks, which show up in this paper.

If as I suspect traditional QCD calculations have missed any essential details, it is in a di quark condensate as a template [11] for a nucleation of initial states contributing to inflation as viewed by Guth, et al. [12] in their papers. In addition, partly for reasons presented in this document, I also believe that the methodology I tried to refine from Abbot's initial paper, as presented, is useful as a template for synthesis of gravitons. This is also due in part to Axion fields described as a "squeezer" to a di quark condensate [11] [13].

We link an initial di quark configuration to instantons, spalererons, and the early universe. Since spalerons are not well understood, this will necessitate a discussion of spalerons and a thermal bath of temperature T [14]. It is also noteworthy that the bridge between the two potential systems mentioned in this document comes from increasing temperature removing a (1-cos \{phi\}) phase contribution to a tilted potential to what is an eventual typical Guth style quartic potential [15]. Disappearance of axion walls, or their equivalent would be in tandem with increase of temperature, leading to conditions at the start of start of inflation which would at that time, past the first instant of Planck time, match necessary and sufficient conditions as to slow roll potential systems used in early universe cosmology.

We argue all of this will set up conditions for describing in detail an effective cosmological constant contribution to brane world production of gravitons in the early universe. This will be done, assuming that the final input of a cosmological constant is significantly less than what the initial cosmological tilted well potential would give, effectively leading to a large burst of gravitons in an early universe.

Doing this though will necessitate an additional fifth dimension. Usually in mathematics, this is done via the circle group, denoted by $\mathbf{T}$ (or by $\mathbb{T}$ ), is the multiplicative group of all complex numbers with absolute value 1 . The name comes from the fact that these numbers lie on the unit circle in the complex plane.

$$
\mathbb{T}=\{z \in \mathbb{C}:|z|=1\} .
$$

The circle group forms a subgroup of $\mathbf{C}^{\times}$, the multiplicative group of all nonzero complex numbers. Since $\mathbf{C}^{\times}$ is Abelian, it follows that $\mathbf{T}$ is as well.

The notation $\mathbf{T}$ for the circle group stems from the fact that $\mathbf{T}^{n}$ (the direct product of $\mathbf{T}$ with itself $n$ times) is geometrically an $n$-torus. The circle group is then a 1-torus. As electromagnetism can essentially be formulated as a gauge theory on a fiber bundle, the circle bundle, with gauge group $U(1)$. Once this geometrical interpretation is understood, it is relatively straightforward to replace $U$ (1) by a general Lie group. Such generalizations are often called Yang-Mills theories. If a distinction is drawn, then it is that Yang-Mills theories occur on a flat space-time, whereas Kaluza-Klein treats the more general case of curved space-time. The base space of KaluzaKlein theory need not be four-dimensional space-time; it can be any (pseudo-) Riemannian manifold, or even a supersymmetric manifold or orbifold. All this will show up in our discussion of how to relate a Randall-Sundrum effective potential to a four dimensional potential system which incorporate axion walls which collapse as we reach an interval of Planck's time $t_{P}$

This paper will finally conclude with a discussion of the relative merits of a space borne system as discussed by the DETF, like the JDEM project [3] [15] [16], which would then not only help us identify early universe candidates for dark matter-dark energy, but also increase the chance of detecting gravitons. We make this final step by assuming that the dark matter-dark energy contribution is tied into the (1-cos \{phi\}) phase contribution to a tilted phase dependent potential spoken of, earlier, which blends into Guths typical inflationary potential for the reasons mentioned earlier on. Readers may peruse several condensed matter applications of false vacuum nucleation to condensed matter current calculations as to get a flavor of the starting point of the di quark nucleation [17] which will be used later on in this document [18] [19]. One of the key differences in the condensed matter approach from the cosmological one, is that the condensed matter approach explicitly uses the Bogomolnyi in equality as a topological charge vanishing in order to drop the contribution of a least action integral to primarily a quartic potential system [20] [21], while the di quark equivalent system in the initial potential system explicitly appeals to an axion domain wall contribution acting as a squeezer, which is temperature dependent, 
and vanishing in mass as a given temperature radically increases. Aside from that the initial structure bears striking similarities, as being part of Sidney Coleman's false vacuum nucleation [21]. Conceivably though, one could make a crude analogy with baryogenesis arguments though, with the contributions due to di quarks [12] largely vanishing. So the Bogomonyi inequality could be approximately cited, but this has yet to be conclusively proven [21].

In any case, our work is taking a very physical interpretation of what was proved in "The embedding of the spacetime in five dimensions: an extension of Campbell-Magaard theorem" where the statement is made by F. Dahia et al. [22] that "We extend Campbell-Magaard embedding theorem by proving that any n-dimensional semi-Riemannian manifold can be locally embedded in an (n+1)-dimensional Einstein space. We work out some examples of application of the theorem and discuss its relevance in the context of modern higher-dimensional space-time theories”. Our analogy equivalent to embedding is to consider the relative magnitude of an effective potential derived from Randall Sundrum branes in a 5 dimensional setting, which is reduced by employing the circle group to being isomorphic with regards to a four dimensional tilted washboard potential of the sort just mentioned. Doing so if we reject an axion mass temperature dependence will lead, perhaps to a higher five dimensional analogue of the Bogomolnyi inequality [23] being used. This is a detail which we will expand upon later in this document.

\section{Inflation, and the Conundrum It Creates for Dark Matter Searches}

Beginning with Guth's eternal inflation paradigm [12], there are a few issues which need to be reviewed as to the importance of eternal inflation, and how it may be modified by observational data.

Let us now consider how eternal inflation is set up, and how variations in step size as concluded in this paradigm may affect a first order transition alluded to in this specific methodology first introduced by Guth in the 1980s [12]. As stated by Guth, in a recent talk at KITP, UC Santa Barbara [24] there are a few basic assumptions which can be considered as axioms as to the construction of inflation:

1) Quantum fluctuations are important on small scales, if and only if one is working with a static space time (i.e. no expanding universe)

2) For inflating space times, quantum fluctuations are "expanded" to be congruent in magnitude with classical sizes (classical fluctuations)

3) Simple random walk picture: In each time interval of $\Delta t \equiv H^{-1}$, the average field $\varphi$ receives an increment with root means squared, of $\Delta \varphi_{q u}=\frac{H}{2 \cdot \pi}$. This increment is super imposed upon the classical motion, which is downward.

4) Quantum fluctuations are equally likely to move field $\varphi$ "up or down" the well of a "harmonic" style potential.

Those who read the presentation should note the conclusion which is something which raises serious questions: i.e.

5) In equations, the probability of an upward fluctuation exceeds $\frac{1}{e^{3}} \cong \frac{1}{20}$ if

$$
\Delta \varphi_{q u} \approx \frac{H}{2 \cdot \pi}>0.61 \cdot\left|\dot{\varphi}_{c l}\right| \cdot H^{-1} \Leftrightarrow \frac{H^{2}}{\left|\dot{\varphi}_{c l}\right|}>3.8
$$

But

$$
\frac{H^{2}}{\left|\dot{\varphi}_{c l}\right|} \sim \frac{\delta \rho}{\rho} \Rightarrow(\text { Scalar }) \text { density perturbations are of order } \mathrm{O}(1)
$$

6) Guth closes his presentation with a statement to the effect that

"Even if there are $10^{1000}$ vacuum states produced by String theory, then perhaps inflation produces overwhelmingly one preferred type of vacuum states over the other possible types of vacuum states"

The question then arises, How could this preferred type of vacuum state be produced, and by what sort of process? 
Prior attempts to model the early stages of the universe by near ideal gas analogies for so called "super cooling" lead to thermodynamically oriented arguments which we will reproduce here [25], and dissect with commentary as to what is to be expected in the first moments of creation.

To whit, from starting with the assumption that we are restricting ourselves to a non dark matter-dark energy regime of matter to be measured experimentally [13]

$$
\rho_{\text {Density }} \equiv 3 \cdot P_{\text {pressure }} \equiv\left[\frac{\pi^{2}}{30}\right] \cdot N(T) \cdot T^{4}
$$

This is assuming that we are working with a degree of freedom for Fermionic and Bosonic matter contributions of the form [13] (neglecting dark matter and dark energy):

$$
N(T)=N_{\text {Bosonic }}(T)+\frac{7}{8} \cdot N_{\text {Fermionic }}(T) \equiv N_{B}(T)+\frac{7}{8} \cdot N_{F}(T)
$$

This lead to, assuming $M_{P} \sim 10^{19} \mathrm{GeV}$, and a critical temperature for electro weak transitions of

$$
T \equiv T_{c} \sim 250 \mathrm{GeV}
$$

This would lead to

$$
N\left(T_{c}\right) \cong 51.5
$$

As well as a temperature dependent volume behaving as [13]

$$
V=\text { volume }=\frac{5.625 \times 10^{57}}{T^{6}} \cdot \frac{1}{N^{3 / 2}(T)}
$$

The radius of this "volume" is directly proportional to $3 \cdot t$ with the time value as (setting the speed of light $c$ $=1)$ :

$$
t=\text { Time at temperature } T=\frac{1}{4 \pi} \cdot \sqrt{\frac{45}{\pi \cdot N(T)}} \cdot\left(\frac{M_{p}}{T^{2}}\right)
$$

Note that the expressions so put up are highly dependent upon the degree of freedom parameter $N(t)$, while saying little as to how this parameter could vary due to a first order phase transition.

This leads to the $2^{\text {nd }}$ consideration of this document, which is how to put in explicit order calculations as to the order of the electroweak phase transition, which Trodden claims is crucial Trodden presents the following arguments [26]:

For continuous transitions, the associated departure from equilibrium is insufficient to lead to relevant baryon number production. For a first order transition, quantum tunneling occurs about $T=T_{C}$, and nucleation of bubbles of the true vacuum in the sea of false (vacuum) begins.

At a particular temperature below $T=T_{C}$, bubbles just large enough to grow nucleate. These are called critical bubbles, and they expand, eventually filling all of space and completing the transition. This, as Trodden notes leads to significant departures from a thermal equilibrium [26].

The final, and important point Trodden makes is what happens in the immediate aftermath of baryogenesis, there is an alteration of a Higgs field, with the Higgs VEV changing from $\langle\phi\rangle=0$ to $\langle\phi\rangle=v\left(T_{c}\right)$, that there is a suppression of baryogenesis, usually due to what is known as a washout to the asymmetry, Trodden's criteria for avoiding the washout of asymmetry, and for permitting baryogenesis to occur and not be suppressed, is to then have

$$
\frac{\left(\left.\langle\phi\rangle\right|_{T \equiv T_{C}} \equiv v\left(T_{C}\right)\right)}{T_{C}} \geq 1
$$

At this juncture, we wish to address how to incorporate a more accurate reading of phase evolution and the 
minimum requirements of phase evolution behavior in an evolving potential system which permits baryogenesis, and also incorporates dark energy production as well. This then answers the question raised earlier ${ }^{25}$, namely does "inflation produces overwhelmingly one preferred type of vacuum states over the other possible types of vacuum states" so one can have not only baryogenesis, but that we also have inflation as well.

To do this, we start off with an embedding in higher dimensions for answering in what context we may have baryogenesis, and when this baryogenesis ceases to be a dominant factor. In addition, we will also, as a side note, answer the question of graviton production in a brane structure arising in pre Planckian physics cosmology. This in particular is not too different from what Wald and others have argued [27] in, with the difference that we are assuming a potential structure for the regime of physics smaller than a Planck's length $l_{P}$, which is not explicitly presented in their arXIV paper. This potential structure necessitates a fifth dimensional embedding which will be referred to in our next section. We found it convenient to use an effective potential given by RandallSundrum brane world theory as a template for this embedding and will use it to answer several physics issues so concerning us, among them being the scarcity of results of spin two gravitons which may not exist in a realistic sense according to Dyson [4]. As well as certain ways to calculate a more realistic vacuum energy based cosmological constant. This will necessitate explaining why a fifth dimension would help matters.

This investigation is attempting to show that the fifth dimension postulated by Randall-Sundrum theory helps give us an action integral which leads to a minimum physical potential we can use to good effect in determining initial conditions for the onset of inflation. The $5^{\text {th }}$ dimension of the Randall-Sundrum brane world is of the genre [28], for $-\pi \leq \theta \leq \pi$

$$
x_{5} \equiv R \cdot \theta
$$

This lead to an additional embedding structure for typical GR fields, assuming as one may write up a scalar potential "field" with $\phi_{0}(x)$ real valued, and the rest of it complex valued as [28]: WITH c.c. REFERRING TO COMPLEX CONJUGATE.

$$
\phi\left(x^{\mu}, \theta\right)=\frac{1}{\sqrt{2 \cdot \pi \cdot R}} \cdot\left\{\phi_{0}(x)+\sum_{n=1}^{\infty}\left[\phi_{n}(x) \cdot \exp (i \cdot n \cdot \theta)+C . C .\right]\right\}
$$

This scalar field makes its way to an action integral structure which will be discussed later on, which Sundrum used to forming an effective potential. Our claim in this analysis can also be used as a way of either embedding a Bogomolyni inequality, perhaps up to five dimensions [22], or a straight forward reduction in axion mass due to a rise in temperature [29] helped reduced effective potential in this structure, with the magnitude of the Sundrum potential forming an initial condition for the second potential of the following phase transition. Note that we are referring to a different form of the scalar potential, which we will call $\phi$, which has the following dynamic [30] [31]. We explain the justification for a possible pre-Planck time, as given here, in the last appendix, Appendix VI, which is referenced in the conclusion with a long discussion. Suffice to say though that the pre Planck time condition is put in the document as to allow for changing mass of the graviton, which we will bring up in our Appendix VI entry. It is a last appendix entry, due to its contribution to the conclusion of our document.

$$
\begin{aligned}
& \tilde{V}_{1} \rightarrow \tilde{V}_{2} \\
& \tilde{\phi}(\text { increase }) \leq 2 \cdot \pi \rightarrow \tilde{\phi}(\text { decrease }) \leq 2 \cdot \pi \\
& t \leq t_{P} \rightarrow t \geq t_{P}+\delta \cdot t
\end{aligned}
$$

The potentials $\tilde{V}_{1}$, and $\tilde{V}_{2}$ were described in terms of S-S' (solition-antisoliton) di quark pairs nucleating and then contributing to a chaotic inflationary scalar potential system. Here, $m^{2} \approx(1 / 100) \cdot M_{P}^{2}$

$$
\begin{gathered}
\tilde{V}_{1}(\phi)=\frac{M_{P}^{2}}{2} \cdot(1-\cos (\tilde{\phi}))+\frac{m^{2}}{2} \cdot\left(\tilde{\phi}-\phi^{*}\right)^{2} \\
\tilde{V}_{2}(\phi) \propto \frac{1}{2} \cdot\left(\tilde{\phi}-\phi_{C}\right)^{2}
\end{gathered}
$$

We should keep in mind that $\phi_{C}$ in Equation (13a) is an equilibrium value of a true vacuum minimum of 
Equation (3a) after tunneling. In the potential system given as Equation (13a) we see a steadily rising scalar field value which is consistent with the physics of Figure 1. In the potential system given by Equation (13b) we see a reduction of the height of a scalar field which is consistent with the chaotic inflationary potential overshoot phenomena. We should note that $\phi^{*}$ in Equation (13a) is a measure of the onset of quantum fluctuations. Appendix I is a discussion of axion potentials which we claim is part of the contribution of the potential given in Equation (13a) Note that the tilt to the potential given in Equation (13a) is due to a quantum fluctuation. As explained by Guth for quadratic potentials [7],

$$
\phi^{*} \equiv\left(\frac{3}{16 \cdot \pi}\right)^{\frac{1}{4}} \cdot \frac{M_{P}^{\frac{3}{2}}}{m^{\frac{1}{2}}} \cdot M_{P} \rightarrow\left(\frac{3}{16 \cdot \pi}\right)^{\frac{1}{4}} \cdot \frac{1}{m^{\frac{1}{2}}}
$$

This in the context of the fluctuations having an upper bound of

$$
\tilde{\tilde{\phi}}>\sqrt{\frac{60}{2 \cdot \pi}} M_{P} \approx 3.1 M_{P} \equiv 3.1
$$

Here, $\tilde{\tilde{\phi}}>\phi_{C}$. Also, the fluctuations Guth had in mind were modeled via [32]

$$
\tilde{\phi} \equiv \tilde{\tilde{\phi}}-\frac{m}{\sqrt{12 \cdot \pi \cdot G}} \cdot t
$$

\section{Brief Sketch of Randall Sundrum Effective Potential and Its Links to Equation (13a) and Equation (13b)}

The consequences of the fifth dimension mentioned in Equation (10) above show up in a simple warped compactification involving two branes, i.e. a Planck world brane, and an IR brane [28]. The IR brane can be thought of as similar to a Casmir physics plate model, parallel to the usual Universe brane, with a small separation between the two branes. This construction with the physics of this 5 dimensional system allow for solving the hierarchy problem of particle physics, and in addition permits us to investigate the following five dimensional action integral [28].

$$
S_{5}=\int \mathrm{d}^{4} x \cdot \int_{-\pi}^{\pi} \mathrm{d} \theta \cdot R \cdot\left\{\frac{1}{2} \cdot\left(\partial_{M} \phi\right)^{2}-\frac{m_{5}^{2}}{2} \cdot \phi^{2}-K \cdot \phi \cdot\left[\delta\left(x_{5}\right)+\delta\left(x_{5}-\pi \cdot R\right)\right]\right\}
$$

This integral, will lead to the following equation to solve

$$
-\partial_{\mu} \partial^{\mu} \phi+\frac{\partial_{\theta}^{2}}{R^{2}} \phi-m_{5}^{2} \phi=K \cdot \frac{\delta(\theta)}{R}+K \cdot \frac{\delta(\theta-\pi)}{R}
$$

Here, what is called $m_{5}^{2}$ can be linked to Kaluza Klein "excitations" [28] via (for $n>0$ )

$$
m_{n}^{2} \equiv \frac{n^{2}}{R^{2}}+m_{5}^{2}
$$

This uses [33] (assuming $l$ is the curvature radius of $\mathrm{AdS}_{5}$ )

$$
m_{5}^{3} \equiv \frac{M_{P}^{2}}{l}
$$

This is for a compactification scale, for $m_{5} \ll \frac{1}{R}$, and after an ansatz of the following is used [28]:

$$
\phi \equiv A \cdot\left[\exp \left(m_{5} \cdot R \cdot|\theta|\right)+\exp \left(m_{5} \cdot R \cdot(\pi-|\theta|)\right)\right]
$$

We then obtain after a non trivial vacuum averaging [28] 


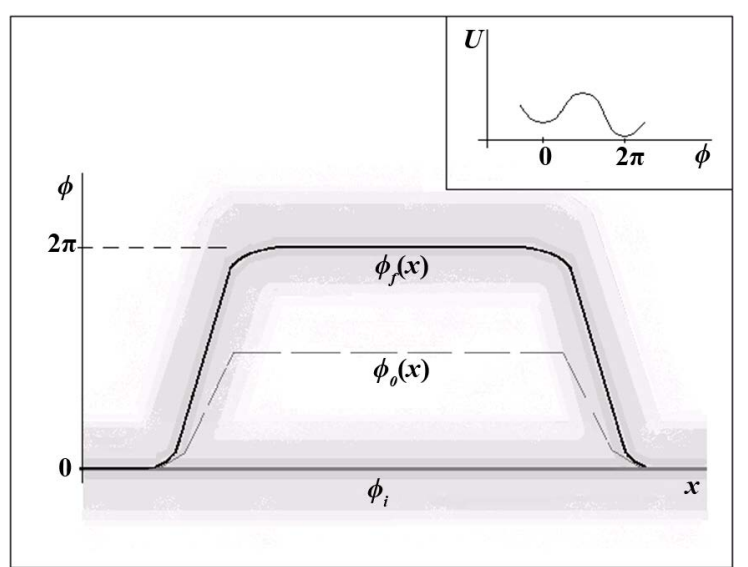

Figure 1. Initial configuration of the domain wall nucleation potential used in Equation (6a) which we claim eventually becomes in sync with Equation (6b) due to the phase transition alluded to by Dr. Edward Kolbs model of how the initial degrees of freedom declined from over 100 to something approaching what we see today in given flat Euclidian space models of space time (i.e. the FRW metric used in standard cosmology).

$$
\begin{gathered}
\langle\phi(x, \theta)\rangle=\Phi(\theta) \\
S_{5}=-\int \mathrm{d}^{4} x \cdot V_{\text {eff }}\left(R_{p h y s}(x)\right)
\end{gathered}
$$

This is leading to an initial formulation of [28]

$$
V_{\text {eff }}\left(R_{\text {phys }}(x)\right)=\frac{K^{2}}{2 \cdot m_{5}} \cdot \frac{1+\exp \left(m_{5} \cdot \pi \cdot R_{\text {phys }}(x)\right)}{1-\exp \left(m_{5} \cdot \pi \cdot R_{\text {phys }}(x)\right)}
$$

Now, if one is looking at an addition of a $2^{\text {nd }}$ scalar term of opposite sign, but of equal magnitude [29]

$$
S_{5}=-\int \mathrm{d}^{4} x \cdot V_{\text {eff }}\left(R_{\text {phys }}(x)\right) \rightarrow-\int \mathrm{d}^{4} x \cdot \tilde{V}_{\text {eff }}\left(R_{\text {phys }}(x)\right)
$$

This is for when we set up an effective Randall-Sundrum potential looking like [29]

$$
\tilde{V}_{\text {eff }}\left(R_{\text {phys }}(x)\right)=\frac{K^{2}}{2 \cdot m_{5}} \cdot \frac{1+\exp \left(m_{5} \cdot \pi \cdot R_{\text {phys }}(x)\right)}{1-\exp \left(m_{5} \cdot \pi \cdot R_{\text {phys }}(x)\right)}+\frac{\tilde{K}^{2}}{2 \cdot \tilde{m}_{5}} \cdot \frac{1-\exp \left(\tilde{m}_{5} \cdot \pi \cdot R_{\text {phys }}(x)\right)}{1+\exp \left(\tilde{m}_{5} \cdot \pi \cdot R_{\text {phys }}(x)\right)}
$$

This above system has a meta stable vacuum for a given special value of $R_{\text {phys }}(x)$ We will from now on use this as a "minimum" to compare a similar action integral for the potential system given by Equation (22) above.

\section{How to Compare the Randall-Sundrum Effective Potential Minimum with an Effective Potential Minimum Involving the Potential of Equation (3) Above}

We are forced to consider two possible routes to the collapse of a complex potential system to the chaotic inflationary model promoted by Guth [7].

The first such model involves a simple reduction of the axion wall potential [13] as given by, especially when $N=1$

$$
V(a)=m_{a}^{2} \cdot\left(f_{P Q} / N\right)^{2} \cdot\left(1-\cos \left[a /\left(f_{P Q} / N\right)\right]\right)
$$

The simplest way to deal with Equation (13) is to set, when Kolb [13] writes 


$$
m_{\text {axion }}(T) \cong 0.1 \cdot m_{\text {axion }}(T=0) \cdot\left(\Lambda_{Q C D} / T\right)^{3.7}
$$

i.e. to declare that the axion "mass" vanishes, and to let this drop off in value give a simple truncated version of chaotic inflationary potentials along the lines given by a transition from Equation (3a) to Equation (3b) We should note that $\Lambda_{Q C D}$ is the enormous value of the cosmological constant which is $10^{120}$ larger than what it is observed to be today [1]-[3], and for now we are side steeping the question of if or not the negative valued Randall-Sundrum cosmological constant [28]

$$
\Lambda_{5}=-\frac{6}{l^{2}}
$$

has a bearing on this situation. Not to mention the problems inherent in several proposed fixes to the cosmological constant problem [34].

Now if we want an equivalent explanation, which may involve baryogenesis, we need to look at the component behavior of each of the terms in Equation (23) without assuming $m_{a}^{2}(T) \underset{T \rightarrow \infty}{\longrightarrow} \varepsilon^{+}$. Then, we need to re define several of the variables presented above. Now, in the typical theory presented by

$$
\frac{M_{P}^{2}}{2} \cdot(1-\cos (\tilde{\phi})) \propto m_{a}^{2} \cdot\left(f_{P Q} / N\right)^{2} \cdot\left(1-\cos \left[a /\left(f_{P Q} / N\right)\right]\right)
$$

We then have to present a varying in magnitude value for the "scalar" $\tilde{\phi}$ involving ultimately the Bogolmolnyi inequality. I have done several of these for condensed matter current problems, but for our cosmology situation, we first have to work with

$$
\left[a /\left(f_{P Q} / N\right)\right] \approx \tilde{\phi}
$$

There has been credible work with instantons in higher dimensions, starting with Hawking's 1999 article [31]. This, however, addresses a way of linking an instanton structure with baryogenesis, dark energy, and issues of how Randall-Sundrum brane structure can be used to formation of initial conditions of inflationary cosmology.

Clarifying what can be done with an instanton style quantum nucleation in multiple dimensions [36] may help us with more acceptable models [6] [37] as to estimating, roughly, a quantum value for the cosmological constant, as an improvement in recent calculations. I refer interested readers to Appendix II on this matter, but for now will restrict this discussion to a qualitative derivation done for condensed matter currents for motivational purposes only. Start with a wave functional

$$
\begin{aligned}
& \Psi \propto \exp \left(-\int \mathrm{d}^{3} x_{\text {space }} \mathrm{d} \tau_{\text {Euclidian }} L_{E}\right) \equiv \exp \left(-\int \mathrm{d}^{4} x \cdot L_{E}\right) \\
& L_{E} \geq|Q|+\frac{1}{2} \cdot\left(\tilde{\phi}-\phi_{0}\right)^{2}\{\} \underset{Q \rightarrow 0}{\longrightarrow} \frac{1}{2} \cdot\left(\tilde{\phi}-\phi_{0}\right)^{2} \cdot\{\}
\end{aligned}
$$

where

$$
\{\}=2 \cdot \Delta \cdot E_{\text {gap }}
$$

This leads, if done correctly to the quadratic sort of potential contribution as given by [37]

$\psi_{\mu}(\varphi) \equiv \psi_{\mu} \cdot \exp \left(\alpha_{\mu} \cdot \varphi^{2}\right)$, At the same time it raises the question of if or not when there is a change from the $1^{\text {st }}$ to the $2^{\text {nd }}$ potential system,

This is for his chaotic inflation model using his potential; I call the $2^{\text {nd }}$ potential

Let us now view a toy problem involving use of a S-S' pair which we may write as [17]-[19]

$$
\tilde{\phi} \approx \pi \cdot\left[\tanh b\left(x-x_{a}\right)+\tanh b\left(x_{b}-x\right)\right]
$$

This is for a di quark pair along the lines given when looking at the first potential system, which is a take off upon Zhitinisky's color super conductor model [11].

\section{Summarizing Different Approaches to Forming a Cosmological Constant}

We should note what a common misconception as to the cosmological constant is. In dimensional terms we often 
see it referred to as a "natural" cosmological constant value in terms of Planck Energy values. This is similar to the problems one observes in a Quantum Field theoretic vacuum summation of zero point energy bosonic fields up to Planck energy values [3].

$$
\Lambda_{\text {natural }} \sim \frac{E_{\text {Plank }}^{4}}{\hbar^{3} \cdot c^{3}} \gg \Lambda_{\text {observed }}
$$

V.G. Gurzadyan, and She-Sheng Lue wrote a world scientific paper [30] giving a derivation to the effect that one can calculate a realistic value for the cosmological constant based upon a wave number based upon a vacuum fluctuation model which gives a Fourier style de composition of vacuum fluctuation wave modes such that if we assume no angular momentum "twisting" and a flat FRW metric

$$
\Lambda=8 \cdot \pi \cdot G \cdot \int \frac{\mathrm{d}^{3} k}{(2 \cdot \pi)^{3}} \cdot\left(\tilde{\varepsilon}(k)=\sqrt{k_{r}^{2}}\right)
$$

Needless to say though, that any energy density so accumulated would be far, far less than what was assumed in the typical bosonic field calculation, above, especially since if $a_{\text {scale }} \equiv$ scale factor size of the universe.

$$
k_{r}=\frac{n \cdot \pi}{a_{\text {scale }}}
$$

This equation would get dramatically smaller for increasing age of the universe to present conditions, with the initial values of it to be similar in "form" to the enormous values of initial energy density outlined above for an initial nucleating universe, especially if we model the initial energy as proportional to the square of Equation (30c) above. This, however, has a serious defect in that it does not give a genesis, or origins reference as to how the cosmological constant could evolve from initial big bang conditions. Mainly due to it being extremely difficult to form $a_{\text {scale }} \equiv$ scale factor size of the universe for initial conditions in the neighborhood of a cosmic singularity for times in the neighborhood of Planck's time $t_{P}$.

Part of the misconception which I think is endemic in this field with respect to forming a cosmological constant which is consistent with known astrophysics observations lies in the difficulty of forming of an effective Field theoretic Hamiltonian for calculation of vacuum energy, i.e. for quasi particles making sense of

$$
\varepsilon_{\text {vacuum }}=\frac{1}{V} \cdot\left\langle H_{Q F T}\right\rangle_{\text {vacuum }}
$$

G.E. Volovick writes a candidate for an acceptable Hamiltonian in this above equation as having a chemical potential addition [3], i.e.

$$
H_{Q F T} \equiv \hat{H}-\mu_{\text {chemical }} \cdot \hat{N}
$$

This assumes that one can actually define a number operator for quasi particles, i.e.

$$
\hat{N}=\int \mathrm{d}^{3} x \cdot \Psi^{*} \cdot \Psi
$$

Again, for early universe conditions, how does one form $\Psi$ for early states of matter? There is a huge literature on this subject, which will be referred to at the end of this document [24] [25] but the wave functionals of the universe ideas, while promising in their own right for tunneling probability conditions for initial nucleation are time INDEPENDENT constructions and do not answer as to changes of initial states of matter-energy very effectively. In addition it is also important to note that initial states of cosmology are being modeled by application of topological defect, and branes very successfully [26] [27]. Still though, if Equation (1d) were actually defined well, we could then start to calculate a field theoretic version of Equation (1b), at least in principle, without having an undetermined at Planck time $t_{P}$ scale factor, as seen in early universe versions of Equation (1c). All these considerations, plus a discussion with Dr. Steinbeck at the UCLA dark matter-dark energy conference in 2006 lead to a review of Abbots hypothesis.

\section{Abbot's 1985 Attempt to Obtain Low Values for the Cosmological Constant}

As of the mid 1980s, Abbot [8] initiated using a tilted washboard potential model for $V(\phi)$ which allegedly 
would permit work with a satisfactory cosmological constant ${ }^{8}$ value based upon a vacuum energy expression given below(with $\lambda_{\text {other }}$ being non a non axion field $\phi$ contribution to total vacuum energy) [8]

$$
\Lambda_{\text {total }}=\lambda_{\text {other }}+V(\phi)
$$

As Abbot admitted though, this model, while giving certain qualitatively attractive features involved an unacceptably long period of final tunneling time based upon [8]

$$
\Gamma \propto M^{4} \cdot \exp (-\tilde{B})
$$

with

$$
\tilde{B} \approx M^{2} f / V_{N}
$$

This idea assumes as Abbot postulated a cascading series of minimum values of $V(\phi)$ for a potential given by

$$
V_{N}(\phi) \equiv M^{4} \cos \left(\frac{\phi_{N}}{2 \cdot \pi \cdot f}\right)+\frac{\epsilon}{2 \cdot \pi \cdot f} \cdot \phi_{N}
$$

Equation (4) lead to a cascading series of local minimum values, where Abbot scaled the local minimum values via setting his scalar field as $\phi_{N}=N$, where $N$ is an integer. It so happens that each minima of Equation (4) had a vacuum density value of

$$
V_{N}=V_{0}+\epsilon
$$

This assumes $V_{0}$ is the vacuum energy of the minimum with the smallest given value of Equation (1g) possible. Also, we assume that $V_{N}-V_{N-1}=\epsilon$.

Typical values for the constants above were $M \sim 1 \mathrm{eV}, f \sim 10^{16} \mathrm{eV}, \in \sim 0.1 \mathrm{eV}$.

This lead to, for final values of tunneling time of the order of $10^{10^{120}}$ [1]-[3] years, for a final cascade value of $V_{N=0}(\phi)$ chosen so that $\Lambda_{\text {total }} \leq \epsilon$ for a value of vacuum energy which was in sync with observed values of a model with realistic cosmological parameters. In particular, it is useful to keep in mind that $\Lambda_{Q C D} \leq 100 \mathrm{MeV}$, and that we are attempting to remove such eccentric values from our calculations. This is in line with a through going construction of a potential system which has ONE transition from a false to a true vacuum, rather than the multiple local minimums Abbot used in his washboard potential model. We expect this will lead to criteria for formation of the escape of gravitons from an early universe brane construction which evolves toward De Sitter space cosmology as a consequence of inflation. In addition we will also address how gravitons could exist, and tie in with the initial production of dark matter-dark energy, while accepting the difficulty of detecting them in post inflationary cosmology models of galaxies, and other strong gravitational centers seen in present day astrophysics.

We looked at Ariel Zhitnitsky's formulation of how to form a condensate of a stable instanton style configuration of cold dark matter as a starting point for how an axion field can initiate forming a so called QCD ball [11] The QCD ball forms instanton configurations which we use to help compute an upper bound to the cosmological constant which we claim is pertinent to the release of gravitons from a brane [28].

Note that in classical GR, potential diagrams have proved useful in analyzing orbits of particles and photons in Schwartzschild geometry. Here, we use the di quark model so formed here as to add in a physically realistic upper bound to forming a cosmological constant input into analyzing when gravitons could be released from an early universe brane. This is similar in part to the use of potential diagrams in investigating Lorentz violations in AdSS $_{5}$ bulk background [29], and we are extending the same methodology to brane motion and graviton motion in $A d S_{5}$ and $A d S S_{5}$. We also add that our method is useful since it is a bridge from a metric with $d S \cong 0$ in the case of initial nucleation of di quark pairs to the more standard $d S \neq 0$ in standard Schwartzshield geometry, while assuming our branes form in $d S \neq 0$.

The important thing to keep in mind, as brought up in a review of an earlier proposal made on this topic is that the di-quark potential satisfies the slow roll condition for inflation. Does one want to have inflation at $100 \mathrm{MeV}$ ? This is not impossible but difficult to achieve and it is necessary for a presenter of any such di quark model how to get the right amplitude of fluctuations [30]. The answer in part to this issue is that scalar field pertubations lead to a fluctuation of the scalar field at the end of inflation which is heavily dependent upon the initial value of 
the potential at the start of di quark nucleation of the scalar field itself. This will be elaborated upon in the conclusion of this document and will constitute part of a follow up of the ideas of this document for future research inquiry. This happens in spite of a transition from a tilted washboard potential to a potential materially similar to Guths quadratic chaotic inflationary potential [7].

We also claim that the process of forming instantons as spoken of in the formation of di quark pairs in itself is useful for the later formation of cold dark matter in the form of QCD (Quantum Chromodynamics) balls, as is elucidated upon later in this document. In addition is the datum that the main part of the potential for forming the single tunneling washboard potential used in the formation of di quark pairs is extremely similar to a cosmic axion potential which is known to have a temperature dependence. This axion potential, as is stated in one of the appendix entries disappears as temperature increases, leading to the more typical Guth chaotic inflationary potential [7] which is then used as a template to fine tune the fluctuation of scalar potential values toward the end of the cosmic inflationary period [7]. We assert that this period of transformation would lead to extensive graviton production in an early universe, as well as dark matter production in a way which is observable via the proposed JDEM satellite system.

\section{Introduction of Spalerons for the Early Universe as a Generalization of Cosmological Instantons for a Re Do of Abbots Procedure}

As referred to in Mukhanov's book on foundations of cosmology, spalerons are a way to introduce motion of a "quasi particle" in a Euclidian metric via use of Wick rotations $\tau=-i t$ Mukhanov introduces two ways for an instanton (spaleron) to have an escape velocity from a rotated Euclidian metric defined potential, in terms of a given thermal bath of temperature $T$. The two limiting cases are in part defined by the formation of an instanton action $S_{I}$, with [14]

$$
T \ll \frac{V\left(q_{m}\right)}{S_{I}} \Rightarrow \text { Rate of escape determined by the instanton }
$$

This assumes

$$
E \ll V(q)
$$

Next

$$
T \gg \frac{V\left(q_{m}\right)}{S_{I}} \Rightarrow \text { Period of oscillation is about zero }
$$

This assumes

$$
E \approx V(q)
$$

When we have the energy of the system close to Equation (35bi), we are in the realm of a first order approximation of escape probability of constituents of a scalar field $\phi$ given by

$$
P \propto \exp \left(-\frac{E_{\text {sphalerons }}}{T}\right)
$$

This assumes that the energy (mass) of a sphaleron is defined via

$$
E_{\text {sphalerons }} \approx V\left(q_{m}\right)
$$

The exact particulars of forming an appropriate instaton $S_{I}$ for a di quark condensate are yet to be satisfactorily determined, but we have candidates for formation of instanton potentials as given as a generalization of Abbots method. While giving this, we will categorically state that the model we are working with assumes a drastic heat up of early nucleation initial conditions to permit after some period of time after a Planck's time $t_{P}$ conditions permitting chaotic inflation. This after the main contribution to the chaotic inflationary potential "tilt" turns from an initial mass contribution to energy, allowing for Guth's quartic potential for scalar fields to be a 
primary contribution to cosmological initial conditions we can measure

\section{Zhnitinisky's QCD Balls, and Their Contribution to Cosmological Instantons}

We looked at Zhitnitsky's formulation of how to form a baryon condensate to initiate forming a so called QCD ball [11]. This QCD ball could in fact be the template for the initial expansion of a scalar field leading to false vacuum inflationary dynamics in the expansion of the universe. Zhitnitsky's formulation uses quarks in a non-hadronic state of matter, but which in the beginning can be in di quark pairs. In doing so, Zhitnitsky's calculations for quarks being squeezed by a so called QCD phase transition due to the violent collapse of an axion domain wall should be used. The axion domain wall would be the squeezer to obtain a soliton (anti soliton) configuration. This pre supposes that we are using a multiple dimensional generalization of the baryon number contribution

$$
M_{B} \approx B^{8 / 9}
$$

And, Zhitnitsky [11] further gives a criteria for absolute stability by writing a region of stability for the QCD balls dependent upon the inequality occurring for $B>B_{C}$ (a critical charge value)

$$
m_{N}>\frac{\partial M_{B}}{\partial B}
$$

He furthermore states that stability, albeit not absolute stability is still guaranteed with

$$
1 \ll B<B_{C}
$$

This is done in such a manner as to use Figure 1 as a condensed matter physics template which is analogous to the instanton behavior of di quark pairs. Furthermore I also claim that it gives a reason for the inclusion of axion physics as seen in the first part of Equation (6a) with respect to the axion walls alluded to in Appendix II below. We should note though that Kolb's “The Early Universe” book [13] also gives a temperature dependence of axions which is of the form of

$$
m_{\text {axion }}(T) \cong 0.1 \cdot m_{\text {axion }}(T=0) \cdot\left(\Lambda_{Q C D} / T\right)^{3.7}
$$

It is important to note that Abbots potential [8] as given above has multiple cascading false vacuum minimum. We are assuming just one minimum, with a final value as given for the first potential system above. Also, if the initial tunneling system is started off with the value of the exponent of Equation (32)

$$
\tilde{B} \approx M^{2} f / V_{N} \rightarrow M^{2} / \tilde{V}_{1}
$$

Then I claim that the tunneling problem Abbot spoke of can be scaled out as to obtain tunneling times not incongruent with respect to a reasonable age of the universe.

We should note that $\Lambda_{Q C D}$ is the enormous value of the cosmological constant which is $10^{120}$ larger than what it is observed to be today. However, if axions are involved in the formation of instanton physics for early universe nucleation, then Equation (40) tells us that as can be expected for very high initial temperatures that axions are without mass but exist as an energy construct, which is not so surprising

Why in this model did I work with a $2^{\text {nd }}$ potential system as well? The reason is because that the first potential system in its emergent fashion is congruent only with the first slow roll condition assumed as necessary for inflation, i.e. if we look at

$$
10^{120}\left(\frac{V_{, \phi}}{V}\right)^{2} \ll 1
$$

We find that $\tilde{V}_{1}$ fits this requirement for small $\phi$ values, but is inconsistent with respect to the following formulation given in Equation (42) below.

$$
\left|\frac{V_{, \phi \phi}}{V}\right| \ll 1
$$


However, if we work with $\tilde{V}_{2}$ that both of these conditions would be amply satisfied. The trick though is to obtain a value of Equation (40) which is not so small as to get the problems Abbot [8] obtained in the mid 1980s which lead to his bound being abandoned due to the effectively infinite time expected in tunneling from a false vacuum minimum to a closer to the general true potential minimum [1] [2].

\section{Estimation of Tunneling Time for New Potential System Given in Equation (13a) and Equation (13b)}

An easy, straightforward manner to calculate tunneling time in the case of a false vacuum is to use a WKB type bounce calculation for forming an energy based tunneling [31]

$$
\tau_{\text {tunneling }} \approx\left|\frac{\partial \cdot S_{W K B}(E)}{\partial|E|}\right|
$$

We need now to do this for a potential system given in part by Equation (5) above, and to do it consistently. Assuming that $S_{\text {WКВ }}(E) \approx S_{I}(E)$ via a Coleman thin wall approximation for a bubble of space time, this leads to [14] [31]

$$
S_{I}(E) \approx \frac{27 \cdot \pi^{2} \cdot \tilde{\sigma}^{4}}{2 \cdot|E|^{3}}
$$

Here, $\tilde{\sigma}$ is the surface tension of a bubble, and

$$
E \equiv V_{\text {min }}
$$

If one defines the minimum of the potential as being due to the $1^{\text {st }}$ tilted washboard potential $E \equiv V_{\min }$ is not going to be a zero quantity, and we will have a non zero but not huge value for tunneling time. This explicitly uses [14]

$$
V\left(\phi^{*}\right) \approx \frac{-3 \cdot \tilde{\sigma}}{R_{\text {crit }}}
$$

If $R_{\text {crit }} \propto l_{P}$, i.e. is on the order of Planck length, and $V\left(\phi^{*}\right) \propto V_{\min }$ of the $1^{\text {st }}$ tilted washboard potential given in Equation (13a), this construction leads to a non zero, but not enormous tunneling time for instantons in the bubble of space time used for an early universe configuration, this will lead to [8]

$$
\left.\Lambda_{\text {total }}\right|_{R_{\text {critical }}}=\lambda_{\text {other }}+V_{\text {min }} \approx \Lambda_{\text {observed }}
$$

This explicitly assumes that one is using the tilted washboard potential of Equation (13a)

\section{Necessary Conditions for Axion Wall Potential Contribution to Equation (13a) Disappearing}

What suffices to initiate chaotic inflation? Note that a potential

$$
V \approx(1 / n) \cdot \phi^{n}
$$

Involves a change of scale factor of the form [14]

$$
a(\phi(t)) \cong a_{\text {init }} \cdot \exp \left(\frac{4 \cdot \pi}{n} \cdot\left(\phi_{\text {init }}^{2}-\phi^{2}(t)\right)\right)
$$

What is involved is a reduction of the degrees of freedom of the initial physical system which permitted the di quark condensate to form in the first place. This would cause the physical system to shift from the $1^{\text {st }}$ to the final Guth chaotic potential [8] form alluded to above which is involving a drop off of higher order terms as seen in Equation (6b) above. Equation (10) if representative of an axion wall contribution to our problem would cease being a factor and largely disappear with increasing temperature, which would be part of the nucleation process. 
As the mass of Equation (40) largely disappeared, this would be in tandem with higher powers of $\mathrm{n}$ disappearing, leading to

$$
V \approx(1 / n) \cdot \phi^{n} \underset{\mathrm{AXION} \rightarrow 0}{\longrightarrow}(1 / 2) \cdot \phi^{2}
$$

This would be in tandem with [14]

$$
a_{\text {init }} \cdot \exp \left(\frac{4 \cdot \pi}{n} \cdot\left(\phi_{\text {init }}^{2}-\phi^{2}(t)\right)\right) \underset{\mathrm{AXION} \rightarrow 0}{\longrightarrow} a_{\text {init }} \cdot \exp \left(\frac{4 \cdot \pi}{2} \cdot\left(\phi_{\text {init }}^{2}-\phi^{2}(t)\right)\right)
$$

\section{Using Our Bound to the Cosmological Constant to Obtain a Conditional Escape of Gravitons from an Early Universe Brane}

In forming this problem, we offer an initial review of the basic physics we are assuming leads up to this problem. The physics shown in Equation (13a) to Equation (13b) above, corresponds to a phase transformation from $d S \cong 0$ in the case of initial nucleation of di quark pairs to the more standard $d S \neq 0$ expected in the mists of to aftermath of inflation. This is where we will use the bound to a cosmological constant given in Equation (40) to determine how we can, address the question of what would be a necessary condition for gravitons to leave an early universe brane, and also to address if they leave an early universe brane, why it is generally going to be extraordinarily difficult to detect gravitons in post inflationary cosmological astrophysical structures [16], such as galaxies. In a nutshell, we believe that the formation of early universe structures at the conclusion of inflation ended the generation of most gravitons, and that it will necessitate a different search methodology in order to obtain graviton signatures experimentally.

Our discussion in part is a brief introduction to the. brane structure built by Leach, and Lesame in their article about escape of gravitons from a brane [46]. It is noticeable that their contribution makes use of an effective potential for gravitons which includes cases where the brane would conceivably move at the same speed as escaping gravitons, and the more desirable case that gravitons moved faster than the brane itself, and escaped to propagate through inter galactic space in the way we see today. Our use of di quarks would be for an emergent structure filling in the bridge between the $1^{\text {st }}$ and $2^{\text {nd }}$ potentials of Equation (5) above, and our analysis of the eventual bound to the cosmological constant is commensurate with an early universe producing most of gravitons we would expect in our present universe. Our candidate for graviton production would be when we are producing cold dark matter in the form of QCD balls as written up by Zhitinisky in his model of QCD color super conductors as a cold dark matter candidate [11]. We also believe that we are tying in this initial period of graviton production with cold dark matter, and that further analysis will lead to refining how to effectively search for gravitons. i.e. the place to start would be in early universe objects, perhaps like quasars.

To begin we present using the paper written by Leach et al. on conditions from a FRW brane [28] in early universe cosmology, assuming that the cosmological constant input is formed as a result of the immediate consequences of the phase transition indicated in Equation (13) above. This also assumes that what we call the cosmological constant was likely initially greater than the final value given for inflationary potentials of the $2^{\text {nd }}$ type given in Equation (13b).

We are assuming explicitly that we can use a Friedmann-Lemaître-Robertson-Walker brane world at or near the end of inflation, and that it is embedded in a structure similar to a 5-dimensional anti-de Sitter Schwartzshield bulk [28]. Why are we doing this? Frankly to utilize the effective graviton potential argument built up by J. Leach et al. as to how a cosmological constant plays into would be release of gravitons from a brane. I believe this criteria is unique, and deserves further analysis.

We shall briefly summarize their findings and put in our work on di quark contributions to an upper bound to the cosmological constant as an enabler of graviton production from this brane structure. A further unstated assumption is that the gravitons were likely released in a brief instant after di quark nucleation. This will be discussed in lieu of the presumed experimental difficulties as to graviton detection via conventional detectors, with recommendations as to how to find gravitons experimentally if they are as I suspect primarily released in early universe inflationary expansion.

Leach et al. obtain a condition for a gravitational signal to be going through a FRW brane via use of an effective potential of a graviton, which is defined via a potential impact parameter [46] 


$$
B^{2}(R)=\frac{f_{k}(R)}{R^{2}}
$$

Also there exists an "impact parameter"

$$
b^{2}=\frac{E^{2}}{P^{2}}
$$

This leads to, practically, a condition of “accessibility” via [28]

$$
b \geq B(R)
$$

Note, that in this definition, $R$ so defined is with respect to "bulk dimensions" and from considerations of a five dimensional anti de Sitter Schwartzshield metric

$$
f_{k}(R)=k+\frac{R^{2}}{l^{2}}-\frac{\mu}{R^{2}}
$$

Here, $k=0$ for flat space, $k=-1$ for hyperbolic three space, and $k=1$ for a three sphere, while we also set an anti-de Sitter radius of curvature [28]

$$
l \equiv \sqrt{\frac{-6}{\Lambda}}
$$

This assumes a negative bulk cosmological constant $\Lambda$ and that $\mu$ is a five dimensional Schwartzshield mass. In addition we also should note that if we assume that the initial configuration of our universe was indistinguishable from a giant black hole and that there exists an effective "radius" for emission of a graviton from a bulk horizon (of super condensed matter-energy) via our standard scale factor $a(t)$

$$
R_{b}(t)=a(t)
$$

Then we have a maximum effective potential of gravitons defined via

$$
B^{2}\left(R_{t}\right)=\frac{1}{l^{2}}+\frac{1}{4 \cdot \mu}
$$

This leads to a bound with respect to release of a graviton from an anti De Sitter brane as defined by Leach et al. as

$$
b \geq B\left(R_{t}\right)
$$

How do we link this to our problem with respect to di quark contributions to a cosmological constant? Here I make several claims.

\section{Claim 1.}

It is possible to re define $l \equiv \sqrt{\frac{-6}{\Lambda}}$ as

$$
l_{\text {eff }}=\sqrt{\left|\frac{6}{\Lambda_{e f f}}\right|}
$$

Here, I am defining $\Lambda_{\text {eff }}$ as being an input from Equation (1) above, with respect to a potential being defined via a mid way transition between the $1^{\text {st }}$ and the $2^{\text {nd }}$ potentials of Equation (5) above. This new value for $l_{\text {eff }}$ is then put into a redefined values for a maximum effective gravitational potential

$$
B_{\text {eff }}^{2}\left(R_{t}\right)=\frac{1}{l_{\text {eff }}^{2}}+\frac{1}{4 \cdot \mu}
$$

\section{Claim 2.}

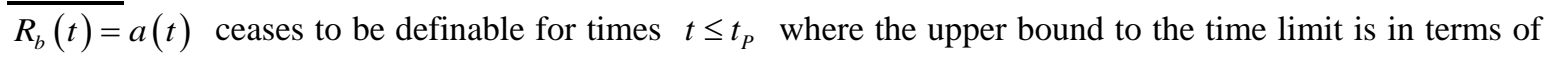


Planck time and in fact the entire idea of a de Sitter, let alone an anti de Sitter metric is not definable in such a physical regime.

\section{Claim 3.}

Equation (5) has a $1^{\text {st }}$ potential which tends to be for a di quark nucleation procedure which just before a defined Planck's time $t_{p}$ permits input into the formation of a cosmological constant according to Equation (40). But that the cosmological constant was prior to time $t_{P}$ likely far higher, perhaps in between the values of the observed cosmological constant of today, and the QCD tabulated cosmological constant which was/is $10^{120}$ time greater. i.e. that there was in fact for the region in between the $1^{\text {st }}$ and the $2^{\text {nd }}$ potential systems of. Equation (5)

$$
b^{2} \geq B_{\text {eff }}^{2}\left(R_{t}\right)=\frac{1}{l_{\text {eff }}^{2}}+\frac{1}{4 \cdot \mu}
$$

With furthermore

$$
\left.\left.\frac{1}{l_{\text {eff }}^{2}}\right|_{t \in t_{P}} \gg \frac{1}{l_{\text {eff }}^{2}}\right|_{t=t_{P}}
$$

So then that there would be a great release of gravitons at or about time $t_{P}$.

\section{Claim 4.}

Few gravitons would be produced significantly after time $t_{P}$. So it would be imperative for researchers to look at early universe objects such as quasars, etc. in order to obtain events consistent with graviton production. We refer to the reader to Appendix $\mathbf{V}$, which summarizes what can be said about gravitons, and early universe physics, with an eye toward distinguishing between different cosmological models.

Before we reach our conclusion, we should note that negative bulk cosmological constant used in brane world with the sort of metric referred to above assumes that there exist positive and negative tensions $\pm \tilde{T}$ with a tension usually given by $\tilde{T}$ becoming $\tilde{T}_{\text {eff }}$ in the revision which was presented as a cosmological bound

$$
\tilde{T}=\frac{3 M_{P}^{2}}{4 \cdot \pi \cdot l} \rightarrow \tilde{T}_{\text {eff }}=\frac{3 M_{P}^{2}}{4 \cdot \pi \cdot l_{\text {eff }}}
$$

Still though this assumes as in Randall-Sundrum brane-worlds that the negative tension brane world has the standard model fields in the negative tension brane [5]. Also that one is able to use a finite distance between the branes to get a discrete KK spectrum, and that a distance between the branes is fixed, to recover standard GR four dimensional space time for low energy systems, which are assumed to be dominant after di quark nucleation.

\section{Connection to Investigating a Tie in of a Brane World Least Action Integral with a Di Quark Least Action Integral}

Now for the question the paper is raising, Can we realistically state the following for initial conditions of a nucleating universe? If so, then what are the consequences?

$$
S_{5}=-\int \mathrm{d}^{4} x \cdot \tilde{V}_{\text {eff }}\left(R_{\text {phys }}(x)\right) \propto\left(-\int \mathrm{d}^{3} x_{\text {space }} \mathrm{d} \tau_{\text {Euclidian }} L_{E}\right) \equiv\left(-\int \mathrm{d}^{4} x \cdot L_{E}\right)
$$

The right hand side of Equation (20) can be stated as having

$$
L_{E} \geq \frac{1}{2} \cdot\left(\tilde{\phi}-\phi_{0}\right)^{2} \cdot\{\}
$$

We can insist that this $\Delta E_{\text {gap }}$ between a false and a true vacuum minimum [21], that

$$
\{\} \equiv 2 \cdot \Delta E_{\text {gap }}
$$

So, this leads to the following question. Does a reduction of axion wall mass for the first potential system given in Equation (13a) being transformed to Equation(13b) above give us consistent physics, due to temperature dependence in axion "mass", or should we instead look at what can be done with S-S' (soliton-anti soliton) in- 
stanton physics and the Bogolmyi inequality [22], in order to perhaps take into account Baryogenesis? Also, can this shed light upon the Wheeler De Witt equations [13] [33] modification by Ashtekar [47] in early universe quantum bounce conditions?

Finally, does this process of baryogenesis, if it occurs lend then to the regime where there is a bridge between classical applications of the Wheeler De Witt equation to the quantum bounce condition raised by Ashtekar [24]?

\section{Tie in with Di Quark Potential Systems, and the Classical Wheeler De-Witt Equation}

Ashtekar's quantum bounce [47] gives a discretized version of the Wheeler De Witt equation. Let us first review classical De Witt theory which incidentally ties in with inflationary $n=2$ scalar potential field cosmology. This will be useful in analyzing consequences of the wave functional so formed in Equation (18) and suggest quantum bounce analogies we will comment upon later.

In the common versions of Wheeler De Witt theory a potential system using a scale radius $R(t)$, with $R_{0}$ as a classical turning point value [13] [33]

$$
U(R)=\left(\frac{3 \cdot \pi \cdot c^{3} \cdot R_{0}}{2 \cdot G}\right)^{2} \cdot\left[\left(\frac{R}{R_{0}}\right)^{2}-\left(\frac{R}{R_{0}}\right)^{4}\right]
$$

Here we have that if $l_{P}$ is a Planck length

$$
R_{0} \sim c \cdot t_{0} \equiv l_{P} \equiv c \cdot \sqrt{\frac{3}{\Lambda}} \sim 7.44 \times 10^{-36} \text { meters }
$$

As well as

$$
\sqrt{\frac{3}{\Lambda}} \equiv t_{P} \sim 2.48 \times 10^{-44} \mathrm{sec}
$$

Now, Henriques [32] presents a way in which one can obtain a Wheeler De Witt equation based upon

$$
\tilde{\hat{H}} \cdot \Psi(\phi)=\left[\frac{1}{2} \cdot\left(A_{\mu} \cdot p_{\phi}^{2}+B_{\mu} \cdot m^{2} \cdot \phi^{2}\right) \cdot \Psi(\phi)\right]
$$

Using a momentum operator as give by

$$
\hat{p}_{t}=-i \cdot \hbar \cdot \frac{\partial}{\partial \cdot \phi}
$$

This is assuming a real scalar field $\phi$ as well as a "scalar mass $m$ " based upon a derivation originally given by Thieumann [48]. The above equation as given by Theumann [48], and secondarily by Henriques [32] lead directly to considering the real scalar field $\phi$ as leading to a prototype wave functional for the $\phi^{2}$ potential term as given by

$$
\psi_{\mu}(\phi) \equiv \psi_{\mu} \cdot \exp \left(\alpha_{\mu} \cdot \phi^{2}\right)
$$

As well as an energy term

$$
\begin{aligned}
& E_{\mu}=\sqrt{A_{\mu} \cdot B_{\mu}} \cdot m \cdot \hbar \\
& \alpha_{\mu}=\sqrt{B_{\mu} / A_{\mu}} \cdot m \cdot \hbar
\end{aligned}
$$

This is for a "cosmic" Schrodinger equation as given by

$$
\tilde{\hat{H}} \cdot \psi_{\mu}(\phi)=E_{\mu}(\phi)
$$


This has

$$
A_{\mu}=\frac{4 \cdot m_{p}}{9 \cdot l_{p}^{9}} \cdot\left(V_{\mu+\mu_{0}}^{1 / 2}-V_{\mu-\mu_{0}}^{1 / 2}\right)^{6}
$$

And

$$
B_{\mu}=\frac{m_{p}}{l_{p}^{3}} \cdot\left(V_{\mu}\right)
$$

Here $V_{\mu}$ is the eigenvalue of a so called volume operator, and the interested readers are urged to consult with the cited paper [48] to go into the details of this, while at the time noting $m_{P}$ is for Planck mass, and $l_{P}$ is for Planck length, and keep in mid that the main point made above, is that a potential operator based upon a quadratic term leads to a Gaussian wave functional with an exponential similarly dependent upon a quadratic $\phi^{2}$ exponent. We do approximate solitons via the evolution of Equation (64) and Equation (64c) above, and so how we reconcile higher order potential terms in this approximation of wave functional is extremely important.

Now Ashtekar in his longer arXIV article [47] make reference to a revision of this momentum operation along the lines of basis vectors $|\mu\rangle$ by

$$
\hat{p}_{\imath}|\mu\rangle=\frac{8 \cdot \pi \cdot \gamma \cdot l_{P}^{2}}{6} \cdot \mu|\mu\rangle
$$

With the advent of this re definition of momentum we are seeing what Ashtekar works with as a sympletic structure with a revision of the differential equation assumed in Wheeler-De Witt theory to a form characterized by [47]

$$
\frac{\partial^{2}}{\partial \phi^{2}} \cdot \Psi \equiv-\Theta \cdot \Psi
$$

$\Theta$ in this situation is such that

$$
\Theta \neq \Theta(\phi)
$$

Also, and more importantly this $\Theta$ is a difference operator, allowing for a treatment of the scalar field as an "emergent time", or "internal time" so that one can set up a wave functional built about a Gaussian wave functional defined via

$$
\max \tilde{\Psi}(k)=\left.\tilde{\Psi}(k)\right|_{k \equiv k^{*}}
$$

This is for a crucial "momentum" value

$$
p_{\phi}^{*}=-\left(\sqrt{16 \cdot \pi \cdot G \cdot \hbar^{2} / 3}\right) \cdot k^{*}
$$

and

$$
\phi^{*}=-\sqrt{3 / 16 \cdot \pi G} \cdot \ln \left|\mu^{*}\right|+\phi_{0}
$$

Which leads to, for an initial point in "trajectory space" given by the following relation $\left(\mu^{*}, \phi_{0}\right)=$ (initial degrees of freedom [dimensionless number] as proportional to an eigenvalue of "momentum", initial "emergent time")

So that if we consider eignfunctions of the De Witt (difference) operator, as contributing toward

$$
e_{k}^{s}(\mu)=(1 / \sqrt{2}) \cdot\left[e_{k}(\mu)+e_{k}(-\mu)\right]
$$

With each $e_{k}(\mu)$ an eignfunction of Equation (65a) above, we have a potentially numerically treatable early universe wave functional data set which can be written as 


$$
\Psi(\mu, \phi)=\int_{-\infty}^{\infty} \mathrm{d} k \cdot \tilde{\Psi}(k) \cdot e_{k}^{s}(\mu) \cdot \exp [i \omega(k) \cdot \phi]
$$

This equation above has a "symmetry" as seen in Figure 1 of Ashtekar's PRL article [47] about $\phi$, reflecting upon a quantum bounce for a pre ceding universe prior to the "big bang" contracting to the singularity and a "rebirth" as seen by a different "branch of Equation (66b) emerging.

\section{Conclusion}

Our model is in part a response to an assertion by Dr. Steinbeck about the feasibility of information exchange in a cyclic universe model. As stated in Appendix III, [24], there is for standard spherically symmetric metrics no prior information exchanged between prior and the present day universe expansion and contraction cycles. What we have formulated so far in this paper is a start toward analyzing initial conditions permitting a cosmological constant without the huge $10^{120}$ magnitude overshoot of current QCD vacuum state values which are reported all over the literature without using his cyclic universe model. QCD balls [11] are a way to introduce a dark matter candidate in directly for formulating this problem and do permit, up to a point adherence to the false vacuum state to a true vacuum value, as postulated by Coleman [20].

Furthermore, we also argue that the semi classical analysis of the initial potential system as given by Equation (6) above and its subsequent collapse is de facto evidence for a phase transition to conditions allowing for cold dark energy to be created at the beginning of inflationary cosmology. And that this production of dark energy would be at the same time as a surge in early universe graviton production this is done with Zhitinisky's QCD ball condensate construction [11] as a multiple dimensional generalization of instanton physics.

Any further work in analyzing this problem will require reconciling the modification of Abbot's hypothesis stated above with what is manifestly wrong in the ideas presented in standard quantum chromodynamics references [1] and furthermore it permits reformulating standard initial conditions for Wheeler-De Witt equation [24] which may permit time dependent effects in the beginning of cosmic nucleation of inflationary universe initial states.

Let us now briefly review what we can say now about standard graviton detection schemes. As mentioned earlier, Rothman states that the Dyson seriously doubts we will be able to detect gravitons via present detector technology [4]. The conundrum is as follows, namely if one defines the criterion for observing a graviton as

$$
\frac{f_{\gamma} \cdot \sigma}{4 \cdot \pi} \cdot\left(\frac{\alpha}{\alpha_{g}}\right)^{3 / 2} \cdot \frac{M_{s}}{R^{2}} \cdot \frac{1}{\varepsilon_{\gamma}} \geq 1
$$

Here,

$$
f_{\gamma}=\frac{L_{\gamma}}{L}
$$

This has $\frac{L_{\gamma}}{L}$ a graviton sources luminosity divided by total luminosity and $R$ as the distance from the graviton source, to a detector. Furthermore, $\alpha=e^{2} / \hbar$ and $\alpha_{g}=G m_{p}^{2} / \hbar$ are the fine structure and gravitational fine structure constants respectively, while $\varepsilon_{\gamma}$ is the hypothesized graviton potential energy. As stated in the manuscript, the problem then becomes determining a cross section $\sigma$ for a graviton production process and $f_{\gamma}=\frac{L_{\gamma}}{L}$.

My entire point is that Dysons conjecture will be true for most candidates for graviton production, but that if we can define early universe graviton production more succinctly via a refinement of the above presented arguments that in due time, most likely by space detection systems looking for dark energy, that we may in fact be able to identify gravitons from an early universe environment. In addition, how the tunneling occurs should be investigated further via a refinement of the bounce solutions and vacuum tunneling procedures used by J. C. Hackworth and E.J. Weinberg in October 2004 [44].

We can point out via a heuristic argument a linkage between the di quark scalar field and fluctuations of a 
scalar field toward the end of the inflationary era which we do believe will support a proposal for refining this inquiry as to getting appropriate data sets from planned JDEM space borne instrument platforms. As pointed out by Zhitinisky [11], in 2002, di quark condensates are a good candidate for the formation of cold dark matter. We can relate amplitude fluctuations from the formation of di quark scalar fields to later fluctuations via the following procedure.

Let us state for the beginning of the inflationary era, where the di quark pairs were already synthesized an amplitude for admissible fluctuations to be written as reaching a peak value when

$$
k \approx H_{i} \cdot a_{i}
$$

This assumes that $H_{i}$ is an initial invariant value of the Hubble constant formed at or about an initial Planck time $t_{P}$, with the $1^{\text {st }}$ potential of Equation (13a) already utilized to obtain the di quark nucleation value of a scalar potential. Here, $a_{i}$ is the initial scale factor. As stated in a description of assumptions given in the bridge between the $1^{\text {st }}$ and $2^{\text {nd }}$ potentials of Equation (13a), we end up with the production of prototype QCD ball style instantons during this time which would eventually be blended into the quadratic $2^{\text {nd }}$ potential of Equation (13b). So being the case we would get a metric fluctuation value

$$
\delta_{\Phi} \approx m \cdot \ln \left(\lambda_{p h} \cdot H_{k}\right)
$$

This assumes we define

$$
\lambda_{p h} \sim a\left(t_{f}\right) \cdot k
$$

This is assuming $k$ so defined is given by Equation (69) above, while

$$
H_{k} \sim H\left(t_{f}\right)
$$

This last equation represents the value of the Hubble parameter at the end of the inflationary epoch, with $t_{f} \gg t_{P}$. We assume when doing this an energy scale

$$
\varepsilon \sim m^{2} \sim 10^{-12} \cdot \varepsilon_{\text {Planck }}
$$

This leads to fluctuations for a quadratic potential as assumed by Guth

$$
\delta_{\Phi}^{\mathrm{MAX}} \approx m \cdot \ln \left(a_{f} / a_{i}\right) \sim m \cdot \phi_{i}^{2}
$$

As Mukhanov [14] states, which is crucial, if the initial scale of the scalar potential $\phi_{i}$ is larger than $m^{-1 / 2}$ then inhomogeneity of the scale $\lambda_{p h} \sim H_{i}^{-1} \cdot a / a_{i}$ become very large before the end of inflation [14]. This conceivably

could be detected in the $f_{\gamma}=\frac{L_{\gamma}}{L}$ value used as an input for Equation (26). In any case though, as raised in the beginning of the manuscript, a base line requirement as to the development of realistic inflationary models, tying into the amplitude of the initial inflationary model is that for inflation to be on going, due to a di quark nucleation at the beginning, with a mass $m$ defined in part by Equation (32)

$$
\phi_{i}>m^{-1 / 2}
$$

All this needs to be vetted and confirmed by space borne observations.

A final consideration to be investigated, for the sake of how we define scale factors lies in an issue that Ellis raised recently in "On Horizons and the Cosmic Landscape" [43]. His point was about the problems of vetting the multi universe hypothesis put in by Susskind. His most cogent point was in defining the existence of an event horizon (assuming that we could consider an initial universe construction as analogous to a black hole) at an arbitrary time $t_{0}$ if either we see convergence of the integral

$$
\int_{t_{0}}^{\infty} \frac{\mathrm{d} t}{a(t)}<\infty \Rightarrow \text { The universe expands forever in the future }
$$




$$
\int_{t_{0}}^{t_{\mathrm{FINAL}}} \frac{\mathrm{d} t}{a(t)}<\infty \Rightarrow \text { The universe comes to an end at a future time, as } a(t) \rightarrow 0
$$

If as I assert that we can not realistically define $a(t)$ for time values in the neighborhood of a Planck time interval, after di quark nucleation and the synthesis of the $1^{\text {st }}$ potential in Equation (5), we have to consider that there is a total wipe out of information at the start of nucleation of a new universe, and that we cannot appeal to the multi universe hypothesis in order to explain via an anthropic principle selection criteria for how our physical constants came into being as they are, with a surviving universe, as we see it today. The last two appendix entries, Appendix IV, and Appendix V, deal with, first (Appendix IV) what is the WKB approximation, with the understanding that we are improving on the WKB approximation, and Appendix $\mathbf{V}$, which elaborates upon how the eventual detection of gravitational waves will allow us to distinguish among different gravitational theories, with a suggestion as to how to improve upon the ideas brought up in section IV. Furthermore we also are considering elaborations as to what is brought up in Appendix VI, which outlines the importance of Pre-Planckian space-time contributions to the physics which we model in Equation (12) and right afterwards, which is instrumental in terms of giving conditions in modeling cosmology, so as to allow for experimental proofs of the di-quark hypothesis, as presented in our document. The Appendix IV and Appendix VI entries should be further developed and inter related in future documents as to turn our suppositions into falsifiable experimental science. Doing so will allow examining Corda's suppositions [37] as part of experimental cosmology and establish once and for all if General relativity is the definitive theory for cosmological evolution, or if Scalar-tensor models have to be invoked to understand the CMBR spectra [45].

While checking Dr. Corda's supposition [37], we will need to use the techniques and instruments as cited in [38] as to the most efficient way to perform appropriate data analysis, so as to investigate if we can experimentally confirm the relevance of General relativity to early universe conditions, Dr. Corda's choice of either scalar tensor gravity or traditional general relativity as the causative agent for instrumental data will be have to be investigated via the instrumentation as written up in [38], and care must be taken to avoid the Bicep 2 disaster, which was due to dust from galaxies, which effectively generated multiple sources. We want a single causative agent as to relic gravitational waves, for our data.

\section{Acknowledgements}

This work is supported in part by National Nature Science Foundation of China grant No. 11375279.

In addition: the author wishes to thank Dr. Steinhardt for his elucidation of the importance of the Abbot model in the UCLA 2006 dark matter convention. Previously, the author had no awareness of this work, or of its deep significance as to parameterization of a realistic value to the cosmological constant. In addition, several researchers at the Kalvi institute Eastern gravity conference in March 2006, noticeably Dr. Thorsten Battefeld stated that much of what I presented was a re hash of Guth and Weinberg's inflationary models, which forced me to enunciate graviton production and detection as crucially important. Finally, reviewers in South Africa pointed out the necessity of linking inflationary magnitude to a given specific upper bound which I claim in part is partly answered in the conclusion leading up to Equation (34) above.

In addition, Dr. Xi Yang is thanked for motivating me to continue physics, in spite of every hardship put my way.

\section{References}

[1] Brown, L.S. (1992) Quantum Field Theory. Cambridge University Press, Cambridge. http://dx.doi.org/10.1017/CBO9780511622649

[2] Carrol, S., Press, W.H. and Turner, E.L. (1992) The Cosmological Constant. Annual Review of Astronomy and Astrophysics, 30, 499-542. http://dx.doi.org/10.1146/annurev.aa.30.090192.002435

[3] Volovik, G. (2006) Vacuum Energies: Myths and Reality. International Journal of Modern Physics D, 15, 1987-2010. http://dx.doi.org/10.1142/S0218271806009431

[4] Rothman, T. and Boughn, S. (2006) Can Gravitons Be Detected? Foundations of Physics, 36, 1801-1825. http://dx.doi.org/10.1007/s10701-006-9081-9

[5] Maartens, R. (2005) Brane-World Cosmology. In: Papantonopoulos, E., Ed., The Physics of the Early Universe, Lecture Notes in Physics, Vol. 653, Springer Verlag, Berlin, 213-249. 
[6] In the D4-D5-E6-E7-E8 VoDou Physics Model, Gravity and the Cosmological Constant Come from the MacDowell-Mansouri Mechanism and the 15-dimensional Spin(2,4) = SU(2,2) Conformal Group.

[7] Guth, A.H. and Weinberg, E. (1983) Could the Universe Have Recovered from a Slow First-Order Phase Transition? Nuclear Physics B, 212, 321-364. http://dx.doi.org/10.1016/0550-3213(83)90307-3

[8] Abbot, L. (1985) A Mechanism for Reducing the Value of the Cosmological Constant. Physics Letters B, 150, 427-430. http://dx.doi.org/10.1016/0370-2693(85)90459-9

[9] Private Communications with Dr. Steinhardt at the UCLA Dark Matter Conference, 2006.

[10] Presentation Given by Dr. Steinhardt at the UCLA Dark Matter Conference.

[11] Zhitinisky, A.R. (2002) Dark Matter as Dense Color Superconductor. Nuclear Physics B_Proceedings Supplements, 124, 99-102. http://dx.doi.org/10.1016/S0920-5632(03)02087-5

[12] Guth, A. (1981) Inflationary Universe: A Possible Solution to the Horizon and Flatness Problems. Physical Review D, 23, 347-356. http://dx.doi.org/10.1103/PhysRevD.23.347

[13] Kolb, E.W. and Turner, M.S. (1990) The Early Universe. Westview Press, Boulder.

[14] Mukhanov, (2005) Physical Foundations of Cosmology. Cambridge University Press, Cambridge.

[15] Beckwith, A.W. (2006) "How S-S” Di Quark Pairs Signify an Einstein Constant Dominated Cosmology, and Lead to New Inflationary Cosmology Physics. Electronic Journal of Theoretical Physics, 9, 7-33.

[16] Kadota, K., Dodelson, S., Hu, W. and Steward, E.D. (2005) Precision of Inflaton Potential Reconstruction from CMB Using the General Slow-Roll Approximation. arXIV:astroph/0505185 v1 9 May 2005

[17] Beckwith, A.W. (2006) A New Soliton-Anti Soliton Pair Creation Rate Expression Improving upon Zener Curve Fitting for I-E Plots. International Journal of Modern Physics B, 20, 849-861.

[18] Beckwith, A.W. (2005) Making an Analogy between a Multi-Chain Interaction in Charge Density Wave Transport and the Use of Wave Functionals to Form S-S’ Pairs. International Journal of Modern Physics B, 19, 3683-3703. http://dx.doi.org/10.1142/S0217979205032358

[19] Beckwith, A.W. (2006) An Open Question: Are Topological Arguments Helpful in Setting Initial Conditions for Transport Problems in Condensed Matter Physics? Modern Physics Letters B, 29, 233-243. http://dx.doi.org/10.1142/s0217984906010585

[20] Coleman, S. (1977) The Fate of the False Vacuum. Physical Review D, 15, 2929-2936. http://dx.doi.org/10.1103/PhysRevD.15.2929

[21] Zee, A. (2003) Quantum Field Theory in a Nutshell. Princeton University Press, Princeton, 279-280.

[22] Dahia, F. and Romero, C. (2002) The Embedding of the Space-Time in Five Dimensions: An Extension of Campbell-Magaard Theorem. Journal of Mathematical Physics, 43, 5804-5814. http://dx.doi.org/10.1063/1.1507824

[23] Lee, J. and Sorkin, R.D. (1988) Derivation of a Bogomolnyi Inequality in Fivedimensional Kaluza-Klein Theory. Communications in Mathematical Physics, 116, 353-364. http://dx.doi.org/10.1007/BF01229199

[24] http://online.itp.ucsb.edu/online/strings_c03/guth/pdf/KITPGuth_2up.pdf

[25] Banerjee, B. and Gavai, R.V. (1993) Supercooling and Nucleation in Phase Transitions of the Early Universe. Modern Physics Letters A, 11, 615-622. http://dx.doi.org/10.1142/S0217732396000631

[26] Trodden, M. (1999) The Cosmology-Particle Physics Connection. arXiv:hep-ph/0605284.

[27] Hollands, S. and Wald, R.M. (2002) Comment on Inflation and Alternative Cosmology. arXiv:hep-th/0210001.

[28] Sundrum, R. (2005) In Three Lectures Given in SSI, 2005: The First One Being: Extra Dimensions, by R. Sundrum, Monday, 1 August 2005, 10:30 a.m.-11:30 a.m., The Second One Being: Extra Dimensions, Ctd, by R. Sundrum, Tuesday, 2 August 2005, 10:30 a.m.-11:30 a.m. And the Final One Being Extra Dimensions, Ctd, by R. Sundrum, Wednesday, 3 August 2005, 10:30 a.m.-11:30 a.m.

[29] Beckwith, A.W. (2006) How One Can Use Di Quark Scalar Fields Permitting a Cosmological Constant Permitting Gravitons Escaping from Early Universe Branes. 36th COSPAR Scientific Assembly, Beijing, 16-23 July 2006.

[30] Gurzadyan, G. and Xue, S.-S. (2003) On the Estimation of the Current Value of the Cosmological Constant. Modern Physics Letters A, 18, 561-568. http://dx.doi.org/10.1142/S0217732303008405

[31] Hawkings, S.W. and Real, H.S. (1999) Inflation, Singular Instantons and Eleven Dimensional Cosmology. Physical Review D, 59, Article ID: 023502. http://dx.doi.org/10.1103/PhysRevD.59.023502

[32] Henriques, A.B. (2006) Loop Quantum Cosmology and the Wheeler-De Witt Equation. General Relativity and Gravitation, 38, 1645-1659. http://dx.doi.org/10.1007/s10714-006-0330-1

[33] Dalarsson, M. and Dalarsson, N. (2005) Tensors, Relativity, and Cosmology. Elsevier Press, Amsterdam.

[34] Griffiths, D. (2004) Introduction to Quantum Mechanics. 2nd Edition, Prentice Hall, Princeton. 
[35] http://hitoshi.berkeley.edu/221a/WKB.pdf

[36] Beckwith, A. (2016) Gedanken Experiment for Fluctuation of Mass of a Graviton, Based on the Trace of GR Stress Energy Tensor-Pre Planckian Conditions That Lead to Gaining of Graviton Mass, and Planckian Conditions That Lead to Graviton Mass Shrinking to $10^{-62}$ Grams. Journal of High Energy Physics, Gravitation and Cosmology, 2, 19-24. http://dx.doi.org/10.4236/jhepgc.2016.21002

[37] Corda, C. (2009) Interferometric Detection of Gravitational Waves: The Definitive Test for General Relativity. International Journal of Modern Physics D, 18, 2275-2282. http://arxiv.org/abs/0905.2502 http://dx.doi.org/10.1142/s0218271809015904

[38] Van Den Broeck, C., et al. (2015) Gravitational Wave Searches with Advanced LIGO and Advanced Virgo. http://arxiv.org/pdf/1505.04621v1.pdf

[39] Cowen, R. (2015) Gravitational Waves Discovery Now Officially Dead: Combined Data from South Pole Experiment BICEP2 and Planck Probe Point to Galactic Dust as Confounding Signal. http://www.nature.com/news/gravitational-waves-discovery-now-officially-dead-1.16830

[40] Cowen, R. (2014) Full-Galaxy Dust Map Muddles Search for Gravitational Waves. http://www.nature.com/news/full-galaxy-dust-map-muddles-search-for-gravitational-waves-1.15975

[41] Beckwith, A. (2016) Non Linear Electrodynamics Contributing to a Minimum Vacuum Energy ("Cosmological Constant”) Allowed in Early Universe Cosmology. Journal of High Energy Physics, Gravitation and Cosmology, 2, 25-32. http://dx.doi.org/10.4236/jhepgc.2016.21003

[42] Beckwith, A. (2016) Gedanken Experiment for Degree of Flatness, or Lack of, in Early Universe Conditions. Journal of High Energy Physics, Gravitation and Cosmology, 2, 57-65. http://dx.doi.org/10.4236/jhepgc.2016.21006

[43] Ellis, G. (2006) Comment—On Horizons and the Cosmic Landscape. arXIV astro-ph/0603266 v 215 March, 2006. http://120.52.73.79/arxiv.org/pdf/astro-ph/0603266.pdf

[44] Hackworth, C. and Weinberg, E.J. (2004) Oscillating Bounce Solutions and Vacuum Tunneling in de Sitter Spacetime. Physical Review D, 71, Article ID: 044014.

[45] Dyson, F. (2012) Is a Graviton Detectable? http://publications.ias.edu/sites/default/files/poincare2012.pdf

[46] Leach, J. and Lesame, W. (2005) Conditional Escape of Gravitons from the Brane. arXIV gr-qc/0502109 v3 26 Apr 2005. http://arxiv.org/abs/gr-qc/0502109v4

[47] Ashtekar, A., Pawlowiski, T. and Singh, P. (2006) Quantum Nature of the Big Bang: An Analytical and Numerical Investigation. arXIV gr-qc/0604013. http://arxiv.org/abs/gr-qc/0604013

[48] Thieumann, T. (1998) QSD V : Quantum Gravity as the Natural Regulator of Matter Quantum Field Theories. Classical and Quantum Gravity, 15, 1281-1314. http://arxiv.org/abs/gr-qc/9705019 


\section{Appendix I}

How we show phase evolves in both Equation (13a) and Equation (13b) above

\section{Appendix I A}

First, the physics of how phase evolves due to potential Equation (13b) leading to chaotic inflation.

We shall make a plausibility argument linking the two potentials mentioned above in Equation 13a and Equation 13b. This will be using a slow roll approximation which is de rigor in inflationary cosmology, namely using $\ddot{\phi} \approx 0$ in line with Kolb's treatment of inflationary cosmology. All these approximations will be in an open neighborhood of a unit of Planck time $\left(t_{P}, t_{P}+\delta \cdot t\right)$.

To start off, we will be using a nearly constant Hubble value of $H$ in an early universe nucleation scheme. The reason for doing this will be considering

$$
\dot{\phi}_{0}+\left(\frac{m^{4}}{3 \cdot H}\right) \cdot V_{, \phi} \equiv 0
$$

This will be done in spite of the well known relationship of

$$
H=\left(\frac{\mathrm{d} \ln a}{\mathrm{~d} t}\right) \cong \sqrt{\frac{8 \cdot \pi}{3}} \cdot V(\phi)
$$

Furthermore, as a very rough approximation, we will be setting $\phi^{\bullet} \approx \phi_{C}$ so we rewrite Equation (6b) in this context as

$$
\tilde{V}_{2}(\phi) \propto \frac{1}{2} \cdot\left(\phi-\phi^{*}\right)^{2}
$$

Let us now review how a di quark phase $\phi$ behaves under the influence of the potential given in Equation (1b) above. This assumes that the original form of Equation (13a) stays the same. We also will be reviewing initial fluctuations of the di quark scalar potential $\phi$ used in Equation (6a) via fluctuations we will write as

$$
\phi=\phi_{0}+\tilde{\delta}
$$

Then, what I write as Equation (1) may be viewed as

$$
\frac{\mathrm{d} \phi_{0}}{\mathrm{~d} t}+\frac{2 \cdot m^{4+N}}{3 \cdot H} \cdot\left(\phi-\phi^{*}\right) \cong \tilde{\delta} \cdot\left[\frac{2 \cdot m^{4+N}}{3 \cdot H}\right]
$$

From now on, we will write

$$
\varepsilon^{+}=\tilde{\delta} \cdot\left(\frac{2 \cdot m^{4+N}}{3 \cdot H}\right)
$$

If so we can write Equation (1d) above as

$$
\frac{\mathrm{d} \phi_{0}}{\mathrm{~d} t}+\frac{\varepsilon^{+}}{\tilde{\delta}} \cdot\left(\phi_{0}+\tilde{\delta} \cdot t\right) \cong \varepsilon^{+} \cdot \phi^{*}
$$

We re write this Equation (1f) as

$$
\frac{\mathrm{d} \phi_{0}}{\mathrm{~d} t}+\frac{\varepsilon^{+}}{\tilde{\delta}} \cdot \phi_{0} \cong-\varepsilon^{+} \cdot t+\varepsilon^{+} \cdot \phi^{*} \approx \varepsilon^{+} \cdot \phi^{*}
$$

This leads to a general equation solution of

$$
\frac{\mathrm{d} \phi_{0, G}}{\mathrm{~d} t}+\frac{\varepsilon^{+}}{\tilde{\delta}} \cdot \phi_{0, G} \equiv 0
$$


This leads to an equation for $\phi_{0, G}$ for a physical system influenced by Equation (1b) as:

$$
\phi_{0, G}=\phi_{i} \cdot \exp \left(-\frac{\varepsilon^{+}}{\tilde{\delta}} \cdot t\right)
$$

Next, let us view the particular solution as given by

$$
\phi_{P} \cong \tilde{\delta} \cdot \phi^{*}
$$
by

So, then we can look at the general situation created by the chaotic potential given by Equation (1b), as given

$$
\phi_{0, T}=\phi_{i} \cdot \exp \left(-\frac{\varepsilon^{+}}{\tilde{\delta}} \cdot t\right)+\tilde{\delta} \cdot \phi^{*}
$$

This assumes we may write $\phi_{0, T}=\phi_{i}$ for sufficiently small times. So to first order we can write

$$
\phi_{0, T}=\phi_{i} \cdot\left(1-\frac{\varepsilon^{+}}{\tilde{\delta}} \cdot t\right)+\tilde{\delta} \cdot \phi^{*} \approx \phi_{i} \cdot\left(1-\frac{\varepsilon^{+}}{\tilde{\delta}} \cdot t\right)
$$

This in the very small time limit is similar to what Guth wrote up in the 1980s.

\section{Appendix I B}

\section{When we look at Equation (6a) of the main text}

Next, let us review the far more complicated situation arising with the tilted washboard potential given in Equation (13a) we will write up as, with $A \cong 100 \cdot \Phi$

$$
V(\phi) \approx A \cdot(1-\cos \phi)+\Phi \cdot\left(\phi-\phi^{*}\right)^{2}
$$

This has a derivative value of the form

$$
V(\phi)_{, \phi} \approx A \cdot\left[(\sin \phi)+2 \cdot \frac{\Phi}{A} \cdot\left(\phi-\phi^{*}\right)\right]
$$

Which we write as

$$
V(\phi)_{, \phi} \approx A \cdot\left[\left(\phi-\frac{1}{3 !} \cdot \phi^{3}+\cdots\right)+\frac{1}{50} \cdot\left(\phi-\phi^{*}\right)\right]
$$

Let us first look at the first to third order contributions to this potential, and then get a slow roll equation for evolution of

$$
\dot{\phi}+\frac{m^{4}}{3 \cdot H} \cdot A \cdot\left[\phi \cdot\left(1+\frac{1}{50}\right)-\frac{1}{6} \cdot \phi^{3}\right] \approx \frac{m^{4}}{3 \cdot H} \cdot \frac{A}{50} \cdot \phi^{*}
$$

This has a general solution equation we may write up as

$$
\dot{\phi}_{G}+\frac{m^{4}}{3 \cdot H} \cdot A \cdot\left[\phi_{G} \cdot\left(1+\frac{1}{50}\right)-\frac{1}{6} \cdot \phi_{G}^{3}\right] \approx 0
$$

If we pick an exponential value for the general phase, and solve for $\alpha$

$$
\phi_{G} \equiv \exp \left(-\alpha_{G} \cdot t\right)
$$

Implies, if we set the time unit as $t_{P}$

$$
-\alpha_{G}+\frac{m^{4}}{3 \cdot H} \cdot A \cdot\left[\left(1+\frac{1}{50}\right)-\frac{1}{6} \cdot \exp \left(-2 \cdot \alpha_{G} \cdot t_{P}\right)+\cdots\right] \approx 0
$$


This would lead for very small time intervals

$$
\alpha_{G} \cong \frac{1}{50} \cdot\left[2 \cdot t_{P}-\frac{1}{\left(\frac{m^{4}}{3 \cdot H}\right)}\right]^{-1}
$$

This is almost always negative, since it would require the following for it to be positive, namely

$t_{P}>\frac{1}{2} \cdot\left(\frac{1}{\left(\frac{m^{4}}{3 \cdot H}\right)}\right)$, which will not happen.

If so then for the re do of the washboard potential mentioned, we have an exponentially growing

$$
\phi_{G} \equiv \exp \left(\left|\alpha_{G}\right| \cdot t\right)
$$

A particular solution for this phase for the washboard potential would lead to the cubic equation of the form

$$
\phi_{P}^{3}-6 \cdot\left(1-\frac{1}{50}\right) \cdot \phi_{P}+6 \cdot \phi^{*} \cong 0
$$

In general this leads to the di quark phase $\phi$ increasing under the influence of Equation (2) above.

This allows us to form a bridge between Equation (1b) and Equation (2). This is incidentally in line with the following graphs given in Dr. Kolb's book, namely Figure 8.1 which gives a schematic illustration of an inflationary potential. In addition, Figure 8.7 gives the non barrier version of Figure 8.1, whereas we assume we do have a barrier for tunneling.

\section{Appendix II}

Forming an axion potential term as part of the contribution to Equation (6a)

Kolb's book [13] has a discussion of an Axion potential given in his Equation (10.27)

$$
V(a)=m_{a}^{2} \cdot\left(f_{P Q} / N\right)^{2} \cdot\left(1-\cos \left[a /\left(f_{P Q} / N\right)\right]\right)
$$

Here, he has the mass of the Axion potential as given by $m_{a}$ as well as a discussion of symmetry breaking which occurs with a temperature $T \approx f_{P Q}$. Furthermore, he states that the Axion goes to a massless regime for high temperatures, and becomes massive as the temperature drops. Due to the fact that Axions were cited by Zhitinisky in his QCD ball formation [11], this is worth considering, and I claim that this potential is part of Equation (6a) with the added term giving a tilt to this potential system, due to the role quantum fluctuations play in inflation. Here, $\mathrm{N}>1$ leads to tipping of the wine bottle potential, and $\mathrm{N}$ degenerate $\mathrm{CP}$-conserving minimal values.

\section{Appendix III}

What to expect from typical calculations of vacuum energy in early universe models

As given by Mirjana Dalarsson and Nils Dalarsson [33], we can cite wave function of the universe arguments in order to give a base line as to what constitutes a Plancks length and Planck's time for the initial starting point of inflationary expansion. The radial turning point is usually cited as

$$
R_{0} \sim c \cdot t_{0} \equiv l_{P} \equiv c \cdot \sqrt{\frac{3}{\Lambda}} \sim 7.44 \times 10^{-36} \text { meters }
$$

With a corresponding time length of

$$
\sqrt{\frac{3}{\Lambda}} \equiv t_{P} \sim 2.48 \times 10^{-44} \mathrm{sec}
$$


This however is due to a wave functional argument for the universe which is time independent and depends only on the space time geometry. We do not have a way to include in an evolutionary history of states of matter contributing to these order of magnitude estimates other than a probalistic argument about space time geometry and the matter field content.

The di quark hypothesis, is intended to be a start to giving more definition to the Wheeler-De Whitt equation, for a wave function of the universe $\Psi(R)$ obeying

$$
\frac{\mathrm{d}^{2} \Psi}{\mathrm{d} z^{2}}+Q^{2}(z) \cdot \Psi=0
$$

This is where $\mathrm{z}$ is a dimensionless scale variable and

$$
Q^{2}(z) \equiv a^{2} \cdot\left(z^{2}-z^{4}\right)
$$

And

$$
a=\frac{9 \cdot \pi \cdot c^{5}}{2 \cdot \hbar \cdot G \cdot \Lambda}
$$

The enormous initial energy densities given in forming the bounce radius effectively preclude information exchange in prior universe cycles with our own, at least from the stand point of entropy of information at such high energy values assumed in the formation of our present universe.

\section{Appendix IV. Brief Statement as to the WKB Approximation}

As given by Griffiths [34] we have the following for the WKB approximation, namely that if we are considering a second-order homogeneous linear differential equation that the procedure as given in [35] actually holds, namely as given by Equation (17) of reference [40] which we give as, if E is equal to the Hamiltonian, with

$$
\psi=\frac{c \exp (-i E t / \hbar)}{\sqrt{2 m(E-V)}} \cdot \exp \left[\frac{ \pm i}{\hbar} \cdot \int^{x_{0}} \sqrt{2 m(E-V(\bar{x}))} \cdot \mathrm{d} \bar{x}\right]
$$

This is the solution to what is actually a Hamilton-Jacobi equation. The solution given above, with no requirement that $\mathrm{E}$ be the energy operator of the Schrodinger equation is inherently semi classical. And an approximation used in tunneling problems.

\section{Appendix V. Working with Astrophysical Conundrums}

We take the following discussion as quoted directly from [41] namely

Quote: from [41] we ask that the readers read this directly from the text, and to fill it in, from there. This section 4, from [41], completely fills in the essential background. [39] is also quoted in the below copy from reference [42]. Keep in mind that the references, as given in the below quote are from [42] and that the reader is urged to this section from [41] directly. Here it is.

\section{Re-Examining Relic Gravitational Wave Models as to What Relic Gravitational Waves Could Tell Us about the Origins of the Early Universe. As Given in an Earlier Paper by the Author}

Quoting from [8] we write the following. We reproduce this, because of the centrality of Equation (27) which is basic. It is very noticeable that in [9] we have that the following quote is particularly relevant to consider, in lieu of our results

"Thus, if advanced projects on the detection of GWs will improve their sensitivity allowing to perform a GWs astronomy (this is due because signals from GWs are quite weak) [1], one will only have to look the interferometer response functions to understand if General Relativity is the definitive theory of gravity. In fact, if only the two response functions (2) and (19) will be present, we will conclude that General Relativity is definitive. If the re- 
sponse function (22) will be present too, we will conclude that massless Scalar-Tensor Gravity is the correct theory of gravitation. Finally, if a longitudinal response function will be present, i.e. Equation (25) for a wave propagating parallel to one interferometer arm, or its generalization to angular dependences, we will learn that the correct theory of gravity will be massive Scalar-Tensor Gravity which is equivalent to $f(R)$ theories. In any case, such response functions will represent the definitive test for General Relativity. This is because General Relativity is the only gravity theory which admits only the two response functions (2) and (19) [4] [7]. Such response functions correspond to the two "canonical" polarizations $h+$ and $h \times$. Thus, if a third polarization will be present, a third response function will be detected by GWs interferometers and this fact will rule out General Relativity like the definitive theory of gravity"

We argue that a third polarization in Gravitational waves from the early universe may be detected, if there is proof positive that in the pre Planckian regime that the Corda conjecture [10] as given below, namely if the following analysis is part of our take on relic gravitational waves, is supported by the kinetic energy being larger than the potential energy, namely what if

"The case of massless Scalar-Tensor Gravity has been discussed in [4] [12] with a "bouncing photons analysis" similar to the previous one. In this case, the line-element in the TT gauge can be extended with one more polarization, labelled with $\Phi(t+z)$, i.e. ..."

\section{This ends our recap of the section given in [8] which we think is important.}

What we are arguing for is that the choice of the vacuum energy as given by Equation (27) may give conclusive proof as to satisfy the Corda conjecture and his supposition as to the existence of an additional polarization [9]. We will, in the future try to extend our results so as to determine if Equation (27) either falsifies or supports the existence of a $3^{\text {rd }}$ polarization. Which will be a way to determine the final disposition of GR as THE theory of Cosmology, or open up the possibility of alternate theories. It is an issue which we think will require extreme diligence. While ending our query as to the possible existence of a third polarization we should mention what would be the supreme benefit of our upcoming analysis of Equation (27), namely how to avoid the conflating of dust, with gravitational waves, i.e. the tragic Bicep 2 mistake [10]-[13]

End of quote from reference [41] which answers questions as to cosmological questions as to what is necessary for physics interpretation of both bicep 2, and the relative strength of polarization.

Which indicate if we have a scalar-tensor theory of gravity, or something else as is discussed in [37]. We also need to avoid the problems alluded to in references [39] which are due to Gravitational wave signals generated due to galactic dust, and can be seen to be due to multiple point sources in generated Gravitational wave signatures.

\section{Appendix VI. Why Pre Planck Time is Mentioned at All in Our Document}

What we are referencing in Equation (12) in the main text is a situation for which there is a pre-Planckian space time for emergent phenomena. The Equation (12) is part of using a publication, [41], which would allow the formation of a graviton, with varying mass, which finally settles upon a rest mass of $10^{-62}$ grams after $10^{-62}$ grams. We pick this procedure as part of our also wanting to know how and why there would be a high degree of flat space, as opposed to curved space, right in the aftermath of Planck time, and an emergent space-time construction would be instrumental in terms of understanding the background as given in publication [41]. We submit that [41] gives evidence as to the feasibility and importance of Pre-Planckian space time to understand more fully the dynamics of space-time as well as our model of emergence given in Equation (12). 